\title{
Quasivelocities and symmetries in non-holonomic systems
}

\author{
Anthony M. Bloch ${ }^{\mathrm{a}}$, Jerrold E. Marsden ${ }^{\mathrm{b}}$ and Dmitry V. Zenkov ${ }^{\mathrm{c} *}$ \\ ${ }^{a}$ Department of Mathematics, University of Michigan, Ann Arbor, \\ MI, USA; ${ }^{b}$ Control and Dynamical Systems, California Institute \\ of Technology, Pasadena, CA, USA; ' ${ }^{C}$ Department of Mathematics, \\ North Carolina State University, Raleigh, NC, USA
}

(Received 11 April 2008; final version received 7 November 2008)

\begin{abstract}
This article is concerned with the theory of quasivelocities for non-holonomic systems. The equations of non-holonomic mechanics are derived using the Lagrange-d'Alembert principle written in an arbitrary configuration-dependent frame. The article also shows how quasivelocities may be used in the formulation of non-holonomic systems with symmetry. In particular, the use of quasivelocities in the analysis of symmetry that leads to unusual momentum conservation laws is investigated, as is the applications of these conservation laws and discrete symmetries to the qualitative analysis of non-holonomic dynamics. The relationship between asymptotic dynamics and discrete symmetries of the system is also elucidated.
\end{abstract}

Keywords: Hamel equations; momentum; symmetry

AMS Subject Classifications: 70F25; 37J60; 70H33

\section{Introduction}

Quasivelocities are the velocities of a mechanical system expressed relative to a configuration-dependent frame. The mathematical underpinnings of quasivelocities in texts such as [1] and [2] have not been as developed as is desirable, although their usefulness was clearly demonstrated. The additional clarity provided by the present article should expand the scope of applications and uses of this important concept.

The goal of this article is to develop the use of quasivelocities in the dynamics of non-holonomic systems with symmetry. Key forms of the equations of dynamics including the Hamel equations and the Euler-Lagrange-Poincaré equations are derived, and their relationship to quasivelocities is spelled out. The equations of motion for non-holonomic systems are obtained, and conditions under which an appropriate choice of frame evokes otherwise hidden momentum conservation laws are found. The resulting formalism is utilized in the analysis of the dynamics of some instructive non-holonomic systems including the Chaplygin sleigh and the sleigh coupled to an oscillator.

Said from a slightly different perspective, quasivelocities are the components of a mechanical system's velocity relative to a set of vector fields that span the fibres of the tangent bundle of the configuration space. These vector fields need not be associated with (local) configuration coordinates, so in fact, this article does not

*Corresponding author. Email: dvzenkov@ncsu.edu 
mention 'quasicoordinates', a vacuous concept that has led to some confusion in the literature. A good example of quasivelocities is the set of components of the body angular velocity of a rigid body rotating about a fixed point.

One of the reasons for using quasivelocities is that the Euler-Lagrange equations written in generalized coordinates are not always effective for analyzing the dynamics of a mechanical system of interest. For example, it is difficult to study the motion of the Euler top if the Euler-Lagrange equations (either intrinsically or in generalized coordinates) are used to represent the dynamics. On the other hand, the use of the angular velocity components relative to a body frame pioneered by Euler [3] results in a much simpler representation of dynamics. Euler's approach was further developed by Lagrange [4] for reasonably general Lagrangians on the rotation group and by Poincare [5] for arbitrary Lie groups (see [6] for details and history). Other examples include the use of velocity and angular velocity components relative to a moving frame in the study of dynamics of a rigid body moving on a surface as discussed in $[7,8]$.

Quasivelocities used in [4] and [5] are associated with a group action. Hamel [9] obtained the equations of motion in terms of quasivelocities that were unrelated to a group action on the configuration space. The Hamel equations include both the Euler-Lagrange and Euler-Poincaré equations (e.g. for the rigid body) as special cases.

This article derives the Hamel equations from a variational point of view; that is, it develops the form of the principle of critical action that is equivalent to the Hamel equations. It is then shown how the quasivelocity approach is useful in the treatment of non-holonomic systems with symmetry. In particular, the selection of suitable quasivelocies appropriate to the presence of discrete symmetries is developed along with the application to the qualitative analysis of dynamics.

Non-holonomic systems with symmetry are studied in [10,33] and improved in related references, such as [11]. According to these papers, symmetry and constraints define certain sub-bundles of the tangent bundle of the configuration manifold. As will be shown, it is beneficial to select frames that respect this sub-bundle structure, that is, frames obtained by concatenation of the bases of these sub-bundles. These frames elucidate the momentum equations and conservation laws in the system.

The dynamics of a non-holonomic system with symmetry on the sub-bundle whose fibres are tangent to the group orbits is governed by the momentum equation, an equation that was introduced in [10]. As shown in [12], the momentum equation is important in the energy-based analysis of stability of relative equilibria. In the absence of external dissipation these relative equilibria are often orbitally asymptotically stable in certain directions in the phase system. Related work on this subject includes [13-15]. Additional background and references may be found in [16].

The definition of non-holonomic momentum and the derivation of the momentum equation in [10] relies on the formalism that uses spatial frames. This article utilizes the flexibility of Hamel equations and gives a derivation of the momentum equation in a body frame. In a sense, this is a more natural and straightforward approach since the spatial momentum is almost never conserved in the non-holonomic setting.

The structure of the momentum equation is then used to find conditions under which one has frames that reveal otherwise hidden momentum conservation laws. In some instances the construction of such a frame is algorithmic, although it often leads to implicitly defined frames. In other instances a frame that depends on the group variables in a non-trivial way is necessary for uncovering momentum conservation laws. An example of this latter case is the unbalanced Chaplygin sleigh. 
It is not unusual to have points in the configuration space where the fields that define these frames become linearly dependent. This feature is studied and its utilization in the qualitative analysis of a system's dynamics is discussed.

This article is organized as follows. In Section 2 we review the Hamel equations. In Section 3 non-holonomic systems are briefly discussed and their dynamics is written in the form of constrained Hamel equations. In Section 4 the equations of motion of systems with symmetry are derived using quasivelocities. This derivation differs from that in [10] and in [11] in interesting ways and illustrates the influence of symmetry on the structure of qusivelocities. The non-holonomic momentum conservation and the corresponding frame selection are discussed. In Section 5 non-holonomic systems on Lie groups and their measure-preserving properties are treated. Examples are given in Section 6, where, in particular, momentum conservation in the unbalanced Chaplygin sleigh and integrability of the coupled sleigh-oscillator system are established. Finally, the asymptotic behaviour of momentum conservation laws is studied.

\section{Lagrangian mechanics}

In this section, we review the equations of motion for holonomic systems from the Lagrangian viewpoint.

\subsection{Equations of motion}

\subsubsection{The Euler-Lagrange equations}

A Lagrangian mechanical system is specified by a smooth manifold $Q$ called the configuration space and a function $L: T Q \rightarrow \mathbb{R}$ called the Lagrangian. In many cases, the Lagrangian is the kinetic minus potential energy of the system with the kinetic energy defined by a Riemannian metric on the configuration manifold and the potential energy being a smooth function on $Q$. If necessary, non-conservative forces can be introduced (e.g. gyroscopic forces that are represented by terms in $L$ that are linear in the velocity), but this is not discussed in this article.

In local coordinates $q=\left(q^{1}, \ldots, q^{n}\right)$ on the configuration space $Q$ we write $L=L(q, \dot{q})$. The dynamics is given by the Euler-Lagrange equations:

$$
\frac{\mathrm{d}}{\mathrm{d} t} \frac{\partial L}{\partial \dot{q}^{i}}=\frac{\partial L}{\partial q^{i}}, \quad i=1, \ldots, n .
$$

These equations were originally derived by Lagrange in 1788 by requiring that simple force balance $\boldsymbol{F}=m \boldsymbol{a}$ be covariant, i.e. expressible in arbitrary generalized coordinates. A variational derivation of the Euler-Lagrange equations, namely Hamilton's principle (also called the principle of critical action), came later in the work of Hamilton in 1834/35. For more details see $[6,16]$.

\subsubsection{The Hamel equations}

In this paragraph we introduce the Hamel's equations. In Section 2.3 we derive these equations from a global variational principle, generalizing the reduced principle of critical action for systems with symmetry. 
In many cases the Lagrangian and the equations of motion have a simpler structure when written using so-called non-commuting variables. An example of such a system is the rigid body. Below we develop a general approach that allows one to obtain the Euler-Lagrange equations in such non-commuting variables.

Let $q=\left(q^{1}, \ldots, q^{n}\right)$ be local coordinates on the configuration space $Q$ and $u_{i} \in T Q$, $i=1, \ldots, n$, be smooth independent local vector fields defined in the same coordinate neighbourhood. ${ }^{1}$ The components of $u_{i}$ relative to the basis $\partial / \partial q^{j}$ will be denoted $\psi_{i}^{j}$; that is,

$$
u_{i}(q)=\psi_{i}^{j}(q) \frac{\partial}{\partial q^{j}}, \quad i, j=1, \ldots, n,
$$

where a sum on $j$ is understood.

Let $v=\left(v^{1}, \ldots, v^{n}\right) \in \mathbb{R}^{n}$ be the components of the velocity vector $\dot{q} \in T Q$ relative to the basis $u_{1}, \ldots, u_{n}$, i.e.

$$
\dot{q}=v^{i} u_{i}(q)
$$

then

$$
l(q, v):=L\left(q, v^{i} u_{i}(q)\right)
$$

is the Lagrangian of the system written in the local coordinates $(q, v)$ on the tangent bundle $T Q$. The coordinates $(q, v)$ are the Lagrangian analogues of non-canonical variables in Hamiltonian dynamics.

Given two elements $v, w \in \mathbb{R}^{n}$, define the antisymmetric bracket operation $[\cdot, \cdot]_{q}: \mathbb{R}^{n} \times \mathbb{R}^{n} \rightarrow \mathbb{R}^{n}$ by

$$
[v, w]_{q}=\left[v^{i} u_{i}, w^{j} u_{j}\right](q)
$$

where $[\cdot, \cdot]$ is the Jacobi-Lie bracket of vector fields on $Q$. Therefore, each tangent space $T_{q} Q$ is isomorphic to the Lie algebra $V_{q}:=\left(\mathbb{R}^{n},[\cdot, \cdot]_{q}\right)$. Thus, if the fields $u_{1}, \ldots, u_{n}$ are independent in $U \subset Q$, the tangent bundle $T U$ is diffeomorphic to a Lie algebra bundle over $U$.

The dual of $[\cdot, \cdot]_{q}$ is, by definition, the operation $[\cdot, \cdot]_{q}^{*}: V_{q} \times V_{q}^{*} \rightarrow V_{q}^{*}$ given by

$$
\left\langle[v, \alpha]_{q}^{*}, w\right\rangle \equiv\left\langle\operatorname{ad}_{v}^{*} \alpha, w\right\rangle:=\left\langle\alpha,[v, w]_{q}\right\rangle .
$$

Here $\mathrm{ad}^{*}$ is the dual of the usual ad operator in a Lie algebra; note that in general this operation need not be associated with a Lie group.

Let $u=\left(u_{1}, \ldots, u_{n}\right) \in T Q \times \cdots \times T Q$. For a function $f: Q \rightarrow \mathbb{R}$, define $u[f] \in V_{q}^{*}$ by $u[f]=\left(u_{1}[f], \ldots, u_{n}[f]\right)$, where $u_{i}[f]=\psi_{i}^{j} \partial_{j} f$ is the usual directional derivative of $f$ along the vector field $u_{i}$. Viewing $u_{i}$ as vector fields on $T Q$ whose fibre components equal 0 (that is, taking the vertical lift of these vector fields), one defines the directional derivatives $u_{i}[l]$ for a function $l: T Q \rightarrow \mathbb{R}$ by the formula

$$
u_{i}[l]=\psi_{i}^{j} \frac{\partial l}{\partial q^{j}} .
$$

The evolution of the variables $(q, v)$ is governed by the Hamel equations

$$
\frac{\mathrm{d}}{\mathrm{d} t} \frac{\partial l}{\partial v}=\left[v, \frac{\partial l}{\partial v}\right]_{q}^{*}+u[l] \equiv \operatorname{ad}_{v}^{*} \frac{\partial l}{\partial v}+u[l]
$$


coupled with (2.3). In (2.5), $u[l]=\left(u_{1}[l], \ldots, u_{n}[l]\right)$. If $u_{i}=\partial / \partial q^{i}$, Equations (2.5) become the Euler-Lagrange Equations (2.1). Below, we will derive Equations (2.5) using the principle of critical action. A different approach that makes connections with algebroids is studied in [17].

\subsection{The virtual displacement principle}

A virtual displacement at $q \in Q$ is an element of the tangent space $T_{q} Q$ denoted $\delta q=\left(\delta q^{1}, \ldots, \delta q^{n}\right)$. The principle of virtual displacements states that the equations of motion are determined by the requirement

$$
\left(\frac{\mathrm{d}}{\mathrm{d} t} \frac{\partial L}{\partial \dot{q}^{i}}-\frac{\partial L}{\partial q^{i}}\right) \delta q^{i}=0
$$

The quantities $\delta q^{1}, \ldots, \delta q^{n}$ for a Lagrangian system are independent. Therefore, Equation (2.6) is equivalent to the Euler-Lagrange Equations (2.1)

We now rewrite the virtual displacement principle using the frame $u_{i}, i=1, \ldots, n$. From Equations (2.2)-(2.4) we obtain

$$
\begin{aligned}
& u_{i}[l]=u_{i}\left(L\left(q^{k}, v^{j} \psi_{j}^{k}\right)\right)=\frac{\partial L}{\partial q^{j}} \psi_{i}^{j}+\frac{\partial L}{\partial \dot{q}^{k}} \frac{\partial\left(v^{l} \psi_{l}^{k}\right)}{\partial q^{j}} \psi_{i}^{j}, \\
& \frac{\partial l}{\partial v^{k}}=\frac{\partial}{\partial v^{k}} L\left(q^{i}, v^{j} \psi_{j}^{i}\right)=\frac{\partial L}{\partial \dot{q}^{i}} \psi_{k}^{i} .
\end{aligned}
$$

Denote the components of the virtual displacement $\delta q$ relative to the basis $u_{1}(q), \ldots, u_{n}(q)$ by $w^{i}$ and define the quantities $c_{i l}^{m}(q)$ by

$$
\left[u_{i}, u_{l}\right]=c_{i l}^{m} u_{m}
$$

One finds that

$$
c_{i l}^{m}=\left(\psi^{-1}\right)_{k}^{m}\left[\frac{\partial \psi_{l}^{k}}{\partial q^{j}} \psi_{i}^{j}-\frac{\partial \psi_{i}^{k}}{\partial q^{j}} \psi_{l}^{j}\right] .
$$

Thus, from (2.7),

$$
\left(\left[v_{1}, v_{2}\right]_{q}\right)^{m}=c_{i j}^{m} v_{1}^{i} v_{2}^{j} \quad \text { and } \quad\left([v, \alpha]_{q}^{*}\right)_{j}=c_{i j}^{m} v^{i} \alpha_{m} .
$$

The principle of virtual displacements becomes

$$
\begin{aligned}
\left(\frac{\mathrm{d}}{\mathrm{d} t} \frac{\partial L}{\partial \dot{q}^{j}}-\frac{\partial L}{\partial q^{j}}\right) \delta q^{j} & =\left(\left[\frac{\mathrm{d}}{\mathrm{d} t} \frac{\partial L}{\partial \dot{q}^{j}}\right] \psi_{i}^{j}-\frac{\partial L}{\partial q^{j}} \psi_{i}^{j}\right) w^{i} \\
& =\left(\frac{\mathrm{d}}{\mathrm{d} t}\left[\frac{\partial L}{\partial \dot{q}^{j}} \psi_{i}^{j}\right]-\frac{\partial L}{\partial \dot{q}^{j}} \frac{d \psi_{i}^{j}}{\mathrm{~d} t}-u_{i}[l]+\frac{\partial L}{\partial \dot{q}^{k}} \frac{\partial\left(v^{l} \psi_{l}^{k}\right)}{\partial q^{j}} \psi_{i}^{j}\right) w^{i} \\
& =\left(\frac{\mathrm{d}}{\mathrm{d} t} \frac{\partial l}{\partial v^{i}}-u_{i}[l]+\frac{\partial L}{\partial \dot{q}^{k}}\left[\frac{\partial \psi_{l}^{k}}{\partial q^{j}} \psi_{i}^{j}-\frac{\partial \psi_{i}^{k}}{\partial q^{j}} \psi_{l}^{j}\right] v^{l}\right) w^{i} \\
& =\left(\frac{\mathrm{d}}{\mathrm{d} t} \frac{\partial l}{\partial v^{i}}-u_{i}[l]+\frac{\partial l}{\partial v^{m}}\left(\psi^{-1}\right)_{k}^{m}\left[\frac{\partial \psi_{l}^{k}}{\partial q^{j}} \psi_{i}^{j}-\frac{\partial \psi_{i}^{k}}{\partial q^{j}} \psi_{l}^{j}\right] v^{l}\right) w^{i} \\
& =\left(\frac{\mathrm{d}}{\mathrm{d} t} \frac{\partial l}{\partial v^{i}}-u_{i}[l]+\frac{\partial l}{\partial v^{m}} c_{i l}^{m} v^{l}\right) w^{i}
\end{aligned}
$$


Since the components $w^{i}$ of $\delta q$ are independent, we conclude that the equations of motion are

$$
\frac{\mathrm{d}}{\mathrm{d} t} \frac{\partial l}{\partial v^{i}}=c_{j i}^{m} \frac{\partial l}{\partial v^{m}} v^{j}+u_{i}[l],
$$

which is the coordinate form of Equations (2.5).

Equations (2.8) were introduced in [9] (see also [1] for details and some history).

\subsection{The principle of critical action}

Let $\gamma:[a, b] \rightarrow Q$ be a smooth curve in the configuration space. A variation of the curve $\gamma(t)$ is a smooth map $\beta:[a, b] \times[-\varepsilon, \varepsilon] \rightarrow Q$ that satisfies the condition $\beta(t, 0)=\gamma(t)$. This variation defines the vector field

$$
\delta \gamma(t)=\left.\frac{\partial \beta(t, s)}{\partial s}\right|_{s=0}
$$

along the curve $\gamma(t)$.

Theorem 2.1: Let $L: T Q \rightarrow \mathbb{R}$ be a Lagrangian and $l: T Q \rightarrow \mathbb{R}$ be its representation in local coordinates $(q, v)$. Then, the following statements are equivalent:

(i) The curve $q(t)$, where $a \leq t \leq b$, is a critical point of the action functional

$$
\int_{a}^{b} L(q, \dot{q}) \mathrm{d} t
$$

on the space of curves $\Omega\left(Q ; q_{a}, q_{b}\right)$ in $Q$ connecting $q_{a}$ to $q_{b}$ on the interval $[a, b]$, where we choose variations of the curve $q(t)$ that satisfy $\delta q(a)=\delta q(b)=0$.

(ii) The curve $q(t)$ satisfies the Euler-Lagrange equations

$$
\frac{\mathrm{d}}{\mathrm{d} t} \frac{\partial L}{\partial \dot{q}}=\frac{\partial L}{\partial q} .
$$

(iii) The curve $(q(t), v(t))$ is a critical point of the functional

$$
\int_{a}^{b} l(q, v) \mathrm{d} t
$$

with respect to variations $\delta v$, induced by the variations $\delta q=w^{i} u_{i}(q)$, and given by

$$
\delta v=\dot{w}+[v, w]_{q} .
$$

(iv) The curve $(q(t), v(t))$ satisfies the Hamel equations

$$
\frac{\mathrm{d}}{\mathrm{d} t} \frac{\partial l}{\partial v}=\left[v, \frac{\partial l}{\partial v}\right]_{q}^{*}+u[l]
$$

coupled with the equations $\dot{q}=\langle u(q), v\rangle \equiv v^{i} u_{i}(q)$.

For the early development of these equations see [5] and [9]. 
Proof: The equivalence of (i) and (ii) is proved by computing the variational derivative of the action functional (2.9),

$$
\delta \int_{a}^{b} L(q, \dot{q}) \mathrm{d} t=\int_{a}^{b}\left(\frac{\partial L}{\partial q} \delta q+\frac{\partial L}{\partial \dot{q}} \delta \dot{q}\right) \mathrm{d} t=\int_{a}^{b}\left(\frac{\partial L}{\partial q}-\frac{\mathrm{d}}{\mathrm{d} t} \frac{\partial L}{\partial \dot{q}}\right) \delta q \mathrm{~d} t .
$$

Denote the components of $\delta q(t)$ relative to the basis $u_{1}(q(t)), \ldots, u_{n}(q(t))$ by $w(t)=\left(w^{1}(t), \ldots, w^{n}(t)\right)$, that is,

$$
\delta q(t)=\langle u, w\rangle \equiv w^{i}(t) u_{i}(q(t)) .
$$

To prove the equivalence of (i) and (iii), we first compute the quantities $\delta \dot{q}$ and $\mathrm{d}(\delta q) / \mathrm{d} t$ :

$$
\begin{gathered}
\delta \dot{q}=\delta\left[v^{i}(t) u_{i}(q(t))\right]=\delta v^{i}(t) u_{i}(q(t))+v^{i}(t) \frac{\partial u_{i}}{\partial q^{j}} \delta q^{j}, \\
\frac{\mathrm{d}(\delta q)}{\mathrm{d} t}=\frac{\mathrm{d}}{\mathrm{d} t}\left(w^{i}(t) u_{i}(q(t))\right)=\dot{w}^{i}(t) u_{i}(q(t))+w^{i}(t) \frac{\partial u_{i}}{\partial q^{j}} \dot{q}^{j} .
\end{gathered}
$$

Since $\delta \dot{q}=\mathrm{d}(\delta q) / \mathrm{d} t$, we obtain

$$
\begin{aligned}
\delta v^{i}(t) u_{i}(q(t)) & =\dot{w}^{i}(t) u_{i}(q(t))+v^{k}(t) w^{l}(t)\left(\frac{\partial u_{l}}{\partial q^{j}} \psi_{k}^{j}-\frac{\partial u_{k}}{\partial q^{j}} \psi_{l}^{j}\right)(q(t)) \\
& =\left(\dot{w}^{i}(t)+c_{k l}^{i}(q(t)) v^{k}(t) w^{l}(t)\right) u_{i}(q(t)) ;
\end{aligned}
$$

that is, ${ }^{2}$

$$
\delta v(t)=\dot{w}(t)+[v(t), w(t)]_{q(t)} .
$$

To prove the equivalence of (iii) and (iv), we use the above formula and compute the variational derivative of the functional (2.10):

$$
\begin{aligned}
\delta \int_{a}^{b} l(q, v) \mathrm{d} t & =\int_{a}^{b}\left(\frac{\partial l}{\partial q} \delta q+\frac{\partial l}{\partial v} \delta v\right) \mathrm{d} t \\
& =\int_{a}^{b}\left(\frac{\partial l}{\partial q} w^{i} u_{i}+\frac{\partial l}{\partial v}\left(\dot{w}+[v, w]_{q(t)}\right)\right) \mathrm{d} t \\
& =\int_{a}^{b}\left(u[l] w+\frac{\partial l}{\partial v}[v, w]_{q(t)}-\frac{\mathrm{d}}{\mathrm{d} t}\left(\frac{\partial l}{\partial v}\right) w\right) \mathrm{d} t \\
& =\int_{a}^{b}\left(u[l]+\left[v, \frac{\partial l}{\partial v}\right]_{q(t)}^{*}-\frac{\mathrm{d}}{\mathrm{d} t} \frac{\partial l}{\partial v}\right) w \mathrm{~d} t .
\end{aligned}
$$

This variational derivative vanishes if and only if the Hamel equations are satisfied.

\subsection{Systems with symmetry}

Assume now that a Lie group $G$ acts on the configuration space $Q$. This action is denoted $q \mapsto g q=\Phi_{g}(q)$. Throughout this article we make the assumption that the action of $G$ on $Q$ is free and proper. The quotient space $Q / G$, whose points are the group orbits, is called the shape space. It is known that if the group action is free and proper then shape space is a smooth manifold and the projection map $\pi: Q \rightarrow Q / G$ is a smooth surjective map with 
a surjective derivative $T_{q} \pi$ at each point. The configuration space thus has the structure of a principal fiber bundle. We denote the bundle coordinates $(r, g)$ where $r$ is a local coordinate in the base, or shape space $Q / G$, and $g$ is a group coordinate. Such a local trivialization is characterized by the fact that in such coordinates the group does not act on the factor $r$ but acts on the group coordinate by left translations. Thus, locally in the base, the space $Q$ is isomorphic to the product $Q / G \times G$ and in this local trivialization, the map $\pi$ becomes the projection onto the first factor.

Let $\mathfrak{g}$ denote the Lie algebra of the group $G$. When the configuration space is a principal fiber bundle, some of the vector fields $u_{1}, \ldots, u_{n}$ can be defined globally, as shown below.

Definition 2.2: We say that the Lagrangian is $G$-invariant if $L$ is invariant under the induced action of $G$ on $T Q$.

\subsection{The Euler-Lagrange-Poincaré equations}

Assuming that the vectors $e_{a}(r), a=1, \ldots, k$, form a basis of $\mathfrak{g}=T_{e} G$ for each $r \in Q / G$, we define the frame $e(r)$ by

$$
e(r)=\left(e_{1}(r), \ldots, e_{k}(r)\right) \in \mathfrak{g}^{k} .
$$

Let $\mathcal{A}_{s}$ be a principal connection on the bundle $\pi: Q \rightarrow Q / G$ (see [16] for a review of principal connections). Below, we will discuss how the structure of the Lagrangian can be used for selecting a connection. Tangent vectors in a local trivialization $Q=Q / G \times G$ at the point $(r, g)$ are denoted $(v, w)$. We write the action of $\mathcal{A}_{s}$ on this vector as $\mathcal{A}_{s}(v, w)$. Using this notation, we can write the connection form in the local trivialization as $\mathcal{A}_{s}(v, w)=\operatorname{Ad}_{g}\left(w_{b}+\mathcal{A} v\right)$, where $w_{b}$ is the left translation of $w$ to the identity and, since we are working locally in shape space, we can regard $\mathcal{A}$ as a g-valued one-form on $Q / G$. The connection components $\mathcal{A}_{\alpha}^{a}$ are defined by writing $\mathcal{A} v=\mathcal{A}_{\alpha}^{a} v^{\alpha} e_{a}$.

Define the vector fields $u_{i}$ by

$$
u_{\alpha}=\frac{\partial}{\partial r^{\alpha}}-\mathcal{A}_{\alpha}^{a}(r) L_{g *} e_{a}(r), \quad u_{\sigma+a}=L_{g *} e_{a}(r), \quad \alpha=1, \ldots, \sigma, a=1, \ldots, k .
$$

Thus the fields $u_{\alpha}$ span the horizontal space and the remaining fields $u_{\sigma+a}$ span the vertical space (tangent space to the group orbit) at $q \in Q$. The components of the velocity vector $\dot{q}$ relative to basis (2.12) are

$$
\dot{r}^{\alpha} \quad \text { and } \quad \Omega^{a}=\xi^{a}+\mathcal{A}_{\alpha}^{a} \dot{r}^{\alpha}
$$

where the $\xi^{a}$ are Lie algebra variables. The Lie algebra element $\xi=\xi^{a} e_{a}(r)$ and the group element $g$ are related by the equation $\xi=L_{g-1} * \dot{g} \equiv g^{-1} \dot{g}$

Theorem 2.3: The equations of motion of a system with a G-invariant Lagrangian $L: T Q \rightarrow \mathbb{R}$ are

$$
\begin{gathered}
\frac{\mathrm{d}}{\mathrm{d} t} \frac{\partial l}{\partial \dot{r}}-\frac{\partial l}{\partial r}=-\left\langle\frac{\partial l}{\partial \Omega}, \mathbf{i}_{i} \mathcal{B}+\left\langle\mathbf{i}_{i} \gamma, \mathcal{A}\right\rangle-\left\langle\gamma, \mathbf{i}_{i} \mathcal{A}\right\rangle+\langle\mathcal{E}, \Omega\rangle\right\rangle, \\
\frac{\mathrm{d}}{\mathrm{d} t} \frac{\partial l}{\partial \Omega}=\operatorname{ad}_{\Omega}^{*} \frac{\partial l}{\partial \Omega}+\left\langle\frac{\partial l}{\partial \Omega}, \mathbf{i}_{i} \mathcal{E}\right\rangle, \\
\dot{g}=g\left(\Omega-\mathbf{i}_{r} \mathcal{A}\right) .
\end{gathered}
$$


Equations (2.13) and (2.14) are independent of the group element $g$ and govern the reduced dynamics. The reconstruction equation (2.15) is used to obtain the group dynamics. The terms that appear on the right-hand sides of Equations (2.13)-(2.15) are defined below.

Proof: Using Equation (2.11), we see that

$$
d e=\langle\gamma(r), e\rangle,
$$

where $\gamma$ is a $\mathfrak{g} \otimes \mathfrak{g}^{*}$-valued one-form on $Q / G$. In coordinates we have

$$
\frac{\partial e_{a}}{\partial r^{\alpha}}=\gamma_{a \alpha}^{b}(r) e_{b}(r)
$$

Let $C_{a b}^{c}$ be the structure constants of the Lie algebra $g$ and let $\mathcal{B}_{s}$ be the curvature of the connection $\mathcal{A}_{s}$; that is $\mathcal{B}_{s}(X, Y)=d \mathcal{A}_{s}($ hor $X$, hor $Y$ ), where $X$ and $Y$ are two vector fields. The curvature can be written in the local representation as

$$
\mathcal{B}_{s}\left(\left(v_{1}, w_{1}\right),\left(v_{2}, w_{2}\right)\right)=\operatorname{Ad}_{g}\left(\mathcal{B}\left(v_{1}, v_{2}\right)\right) .
$$

The coordinate representation of $\mathcal{B}$ is $\mathcal{B}\left(v_{1}, v_{2}\right)=\mathcal{B}_{\alpha \beta}^{a} v_{1}^{\alpha} v_{2}^{\beta}$, with $\mathcal{B}_{\alpha \beta}^{a}$ given by the formula

$$
\mathcal{B}_{\alpha \beta}^{c}=\frac{\partial \mathcal{A}_{\alpha}^{c}}{\partial r^{\beta}}-\frac{\partial \mathcal{A}_{\beta}^{c}}{\partial r^{\alpha}}-C_{a b}^{c} \mathcal{A}_{\alpha}^{a} \mathcal{A}_{\beta}^{b}
$$

Define the form $\mathcal{E}$ by

$$
\mathcal{E}=\gamma-\operatorname{ad}_{\mathcal{A}}
$$

which, in coordinates, reads $\mathcal{E}_{\alpha b}^{c}=\gamma_{b \alpha}^{c}-C_{a b}^{c} \mathcal{A}_{\alpha}^{a}$. Straightforward calculation shows that

$$
\begin{aligned}
{\left[u_{\alpha}, u_{\beta}\right] } & =\left(\mathcal{B}_{\alpha \beta}^{c}+\gamma_{b \beta}^{c} \mathcal{A}_{\alpha}^{b}-\gamma_{b \alpha}^{c} \mathcal{A}_{\beta}^{b}\right) u_{\sigma+c}, \\
{\left[u_{\alpha}, u_{\sigma+b}\right] } & =\mathcal{E}_{\alpha b}^{c} u_{\sigma+c}, \\
{\left[u_{\sigma+a}, u_{\sigma+b}\right] } & =C_{a b}^{c} u_{\sigma+c} .
\end{aligned}
$$

Thus, from the definitions of $\mathcal{B}, \mathcal{E}$ and formulae (2.19)-(2.21) one obtains

$$
\begin{aligned}
{\left[\left(\dot{r}_{1}, 0\right),\left(\dot{r}_{2}, 0\right)\right]_{q} } & =\mathcal{B}\left(\dot{r}_{1}, \dot{r}_{2}\right)-\left\langle\mathbf{i}_{\dot{r}_{1}} \gamma, \mathbf{i}_{\dot{r}_{2}} \mathcal{A}\right\rangle+\left\langle\mathbf{i}_{\dot{r}_{2}} \gamma, \mathbf{i}_{\dot{r}_{1}} \mathcal{A}\right\rangle, \\
{[(\dot{r}, 0),(0, \Omega)]_{q} } & =\left\langle\mathbf{i}_{\dot{r}} \mathcal{E}, \Omega\right\rangle, \quad\left[\left(0, \Omega_{1}\right),\left(0, \Omega_{2}\right)\right]_{q}=\operatorname{ad}_{\Omega_{1}} \Omega_{2} .
\end{aligned}
$$

Using (2.22) and evaluating $[\cdot, \cdot]_{q}^{*}$, Equations (2.5) become (2.13)-(2.15).

Now assume that the $G$-invariant Lagrangian equals the kinetic minus potential energy of the system and that the kinetic energy is given by a Riemannian metric $\langle\langle\cdot, \cdot\rangle\rangle$ on the configuration space $Q$.

Definition 2.4: The mechanical connection $\mathcal{A}^{\text {mech }}$ is, by definition, the connection on $Q$ regarded as a bundle over shape space $Q / G$ that is defined by declaring its horizontal space at a point $q \in Q$ to be the subspace that is the orthogonal complement to the tangent space to the group orbit through $q \in Q$ using the kinetic energy metric. The locked inertia tensor $\square(q): \mathfrak{g} \rightarrow \mathfrak{g}^{*}$ is defined by $\langle\square(q) \xi, \eta\rangle=\left\langle\left\langle\xi_{Q}(q), \eta_{Q}(q)\right\rangle\right\rangle$, where $\xi_{Q}$ is the infinitesimal generator of $\xi \in \mathfrak{g}$ and where $\langle\langle\cdot, \cdot\rangle\rangle$ is the kinetic energy inner product. 
Given a system with symmetry, one may use the mechanical connection to set up Equations (2.13) and (2.14). This choice of connection changes the Lie algebra variables from $\xi$ to the local version of the locked angular velocity $\Omega$, which has the physical interpretation of the body angular velocity. This choice also is such that the kinetic energy metric becomes block-diagonal, that is,

$$
\langle\langle\dot{q}, \dot{q}\rangle\rangle=\langle\langle\dot{r}, \dot{r}\rangle\rangle+\langle\langle\Omega, \Omega\rangle\rangle
$$

When the momentum $p=\partial_{\Omega} l$ is used as an independent variable, Equations (2.13) and (2.14) are called the Euler-Lagrange-Poincaré equations. In coordinates, these equations read:

$$
\begin{aligned}
\frac{\mathrm{d}}{\mathrm{d} t} \frac{\partial l}{\partial \dot{r}^{\alpha}}-\frac{\partial l}{\partial r^{\alpha}} & =-\left(\mathcal{B}_{\alpha \beta}^{c}+\left(\gamma_{b \beta}^{c} \mathcal{A}_{\alpha}^{b}-\gamma_{b \alpha}^{c} \mathcal{A}_{\beta}^{b}\right)\right) p_{c} \dot{r}^{\beta}-\mathcal{E}_{\alpha b}^{c} p_{c} \Omega^{b} \\
\dot{p}_{b} & =C_{a b}^{c} p_{c} \Omega^{a}+\mathcal{E}_{\alpha b}^{c} p_{c} \dot{r}^{\alpha}
\end{aligned}
$$

(see also [11]).

Example: The generalized rigid body. If the configuration space is a Lie group $G$ and the Lagrangian is $G$-invariant, Equations (2.13) and (2.14) become

$$
\frac{\mathrm{d}}{\mathrm{d} t} \frac{\partial l}{\partial \xi}=\operatorname{ad}_{\xi}^{*} \frac{\partial l}{\partial \xi} .
$$

These equations are called the Euler-Poincare equations (see [3] $]^{3}$ and [4], Vol. II, for the case $G=\mathrm{SO}(3)$, and [5] for the general case); they describe the momentum dynamics of a generalized rigid body. For more on the history of these equations see [6].

\section{Non-holonomic systems}

\subsection{The Lagrange-d'Alembert principle}

Assume now that there are velocity constraints imposed on the system. We confine our attention to constraints that are homogeneous in the velocity. Accordingly, we consider a configuration space $Q$ and a distribution $\mathcal{D}$ on $Q$ that describes these constraints. Recall that a distribution $\mathcal{D}$ is a collection of linear subspaces of the tangent spaces of $Q$; we denote these spaces by $\mathcal{D}_{q} \subset T_{q} Q$, one for each $q \in Q$. A curve $q(t) \in Q$ will be said to satisfy the constraints if $\dot{q}(t) \in \mathcal{D}_{q(t)}$ for all $t$. This distribution will, in general, be non-integrable; i.e. the constraints are, in general, non-holonomic. ${ }^{4}$

Consider a Lagrangian $L: T Q \rightarrow \mathbb{R}$. In coordinates $q^{i}, i=1, \ldots, n$, on $Q$ with induced coordinates $\left(q^{i}, \dot{q}^{i}\right)$ for the tangent bundle, we write $L\left(q^{i}, \dot{q}^{i}\right)$. The equations of motion are given by the following Lagrange-d'Alembert principle.

Definition 3.1: The Lagrange-d'Alembert equations of motion for the system are those determined by

$$
\delta \int_{a}^{b} L(q, \dot{q}) \mathrm{d} t=0,
$$

where we choose variations $\delta q(t)$ of the curve $q(t)$ that satisfy $\delta q(a)=\delta q(b)=0$ and $\delta q(t) \in \mathcal{D}_{q(t)}$ for each $t$ where $a \leq t \leq b$. 
This principle is supplemented by the condition that the curve $q(t)$ itself satisfies the constraints. Note that we take the variation before imposing the constraints; that is, we do not impose the constraints on the family of curves defining the variation. This is well-known to be important to obtain the correct mechanical equations (see [10] for a discussion and references).

One way to write the dynamics is to make use of the Euler-Lagrange equations with multipliers. This is done below in coordinates.

The distribution $\mathcal{D}$ can be locally written as

$$
\mathcal{D}=\left\{\dot{q} \in T Q \mid A_{i}^{s}(q) \dot{q}^{i}=0, s=1, \ldots, p\right\} .
$$

The constrained variations $\delta q(t) \in T Q$ satisfy the equations

$$
A_{i}^{s}(q) \delta q^{i}=0, \quad s=1, \ldots, p .
$$

Using (2.6) and (3.1), one writes the equations of motion with Lagrange multipliers as

$$
\frac{\mathrm{d}}{\mathrm{d} t} \frac{\partial L}{\partial \dot{q}^{i}}=\frac{\partial L}{\partial q^{i}}+\lambda_{s} A_{i}^{s}(q), \quad A_{i}^{s}(q) \dot{q}^{i}=0 .
$$

\subsection{The constrained Hamel equations}

Given a non-holonomic system, that is, a Lagrangian $L: T Q \rightarrow \mathbb{R}$ and constraint distribution $\mathcal{D}$, select the independent (local) vector fields

$$
u_{i}: Q \rightarrow T Q, \quad i=1, \ldots, n,
$$

such that $\mathcal{D}_{q}=\operatorname{span}\left\{u_{1}(q), \ldots, u_{n-p}(q)\right\}$. Each $\dot{q} \in T Q$ can be uniquely written as

$$
\dot{q}=\left\langle u(q), v^{\mathcal{D}}\right\rangle+\left\langle u(q), v^{\mathcal{U}}\right\rangle, \quad \text { where }\left\langle u(q), v^{\mathcal{D}}\right\rangle \in \mathcal{D}_{q},
$$

i.e., $\left\langle u(q), v^{\mathcal{D}}\right\rangle$ is the component of $\dot{q}$ along $\mathcal{D}_{q}$. Similarly, each $a \in T^{*} Q$ can be uniquely decomposed as

$$
a=\left\langle a_{\mathcal{D}}, u^{*}(q)\right\rangle+\left\langle a_{\mathcal{U}}, u^{*}(q)\right\rangle,
$$

where $\left\langle a_{\mathcal{D}}, u^{*}(q)\right\rangle$ is the component of $a$ along the dual of $\mathcal{D}_{q}$, and where $u^{*}(q) \in T^{*} Q \times \cdots \times T^{*} Q$ denotes the dual frame of $u(q)$. Using (3.2), the constraints read

$$
v=v^{\mathcal{D}} \quad \text { or } \quad v^{\mathcal{U}}=0 \text {. }
$$

This implies

$$
\delta v=\delta v^{\mathcal{D}} \quad \text { or } \quad \delta v^{\mathcal{U}}=0 .
$$

Using the principle of critical action and (3.3) proves the following theorem.

Theorem 3.2: The dynamics of a non-holonomic system is represented by the constrained Hamel equations

$$
\left(\frac{\mathrm{d}}{\mathrm{d} t} \frac{\partial l}{\partial v}-\left[v^{\mathcal{D}}, \frac{\partial l}{\partial v}\right]_{q}^{*}-u[l]\right)_{\mathcal{D}}=0, \quad v^{\mathcal{U}}=0, \quad \dot{q}=\left\langle u(q), v^{\mathcal{D}}\right\rangle .
$$


In coordinate notation, equations in (3.4) read as follows:

$$
\frac{\mathrm{d}}{\mathrm{d} t} \frac{\partial l}{\partial v^{i}}=c_{j i}^{m} \frac{\partial l}{\partial v^{m}} v^{j}+u_{i}[l], \quad \dot{q}=v^{i} u_{i}(q), \quad i, j=1, \ldots, n-p .
$$

\subsection{The Hamel equations with Lagrange multipliers}

Assuming that the distribution $\mathcal{D}$ is locally written as

$$
\mathcal{D}_{q}=\left\{v \in \mathbb{R}^{n} \mid\left\langle a^{s}(q), v\right\rangle=a_{i}^{s}(q) v^{i}=0, s=1, \ldots, p\right\},
$$

and taking into account that variations are written as $\delta q(t)=\langle w(t), u(q(t))\rangle$, we obtain

$$
\langle A(q(t)), \delta q(t)\rangle=\langle a(q(t)), w(t)\rangle .
$$

In the above, $a(q)=\left(a^{1}(q), \ldots, a^{p}(q)\right) \in T_{q}^{*} Q \times \cdots \times T_{q}^{*} Q$. Equation (3.5) implies that the constrained variations of the curve $v(t) \in \mathbb{R}^{n}$ are given by

$$
\delta v(t)=\dot{w}(t)+[v(t), w(t)]_{q(t)} \quad \text { with }\langle a(q(t)), w(t)\rangle=0 .
$$

Therefore,

$$
\delta \int_{a}^{b} l(q, v) \mathrm{d} t=\int_{a}^{b}\left(u[l]+\left[v, \frac{\partial l}{\partial v}\right]_{q}^{*}-\frac{\mathrm{d}}{\mathrm{d} t} \frac{\partial l}{\partial v}+\lambda_{s} a^{s}\right) w \mathrm{~d} t
$$

which implies

$$
\frac{\mathrm{d}}{\mathrm{d} t} \frac{\partial l}{\partial v}-\left[v^{\mathcal{D}}, \frac{\partial l}{\partial v}\right]_{q}^{*}-u[l]=\lambda_{s} a^{s}, \quad\langle a(q), v\rangle=0, \quad \dot{q}=\langle u(q), v\rangle .
$$

If the fields $u_{1}(q), \ldots, u_{n-p}(q)$ span the subspace $\mathcal{D}_{q}$, Equations (3.6) are equivalent to (3.4). We will see in Section 6 that Equations (3.6) are useful when studying the dynamics of the spatial momentum.

Below we evaluate the Lagrange multipliers for systems whose Lagrangian $L: T Q \rightarrow \mathbb{R}$ equals the kinetic minus potential energy, and the kinetic energy is given by a Riemannian metric on $Q$ :

$$
L(q, \dot{q})=\frac{1}{2}\langle\langle\dot{q}, \dot{q}\rangle\rangle-U(q)
$$

Let $G_{q}: T_{q} Q \rightarrow T_{q}^{*} Q$ be the kinetic energy metric; that is, $\langle\langle\dot{q}, \dot{q}\rangle\rangle=\left\langle G_{q} v, v\right\rangle$. Let $\mu=\partial l / \partial v=G_{q} v \in T_{q}^{*} Q$ be the conjugate momentum. The constraints in the momentum representation are given by

$$
\left\langle a(q), G_{q}^{-1} \mu\right\rangle=\langle\mu, b(q)\rangle=0,
$$

where $b(q)=G_{q}^{-1} a(q) \in T_{q} Q$. Using (3.6), we get

$$
\dot{\mu}=\left[v^{\mathcal{D}}, \mu\right]_{q}^{*}+u[l]+\lambda_{s} a^{s} .
$$


Let $\lambda=\left(\lambda_{1}, \ldots, \lambda_{p}\right)$. Taking the time derivative of $\langle\mu, b(q)\rangle=0$ and replacing $\dot{\mu}$ with the right-hand side of (3.7), we obtain

$$
\langle\mu, \dot{b}(q)\rangle+\left\langle\left[v^{\mathcal{D}}, \mu\right]_{q}^{*}+u[l], b(q)\right\rangle+\lambda\langle a(q), b(q)\rangle=0,
$$

which implies

$$
\begin{aligned}
\lambda & =-\left(\langle\mu, \dot{b}(q)\rangle+\left\langle\left[v^{\mathcal{D}}, \mu\right]_{q}^{*}+u[l], b(q)\right\rangle\right) \cdot\langle a(q), b(q)\rangle^{-1} \\
& =-\left(\left\langle\frac{\partial l}{\partial v}, \dot{b}(q)\right\rangle+\left\langle\left[v^{\mathcal{D}}, \frac{\partial l}{\partial v}\right]_{q}^{*}+u[l], b(q)\right\rangle\right) \cdot\langle a(q), b(q)\rangle^{-1} .
\end{aligned}
$$

\section{Dynamics of non-holonomic systems with symmetry}

\subsection{Reduced dynamics}

Assume that a Lie group $G$ acts freely and properly on the configuration space $Q$ and that the Lagrangian $L$ and constraint distribution $\mathcal{D}$ are invariant with respect to the induced action of $G$ on $T Q$.

Although it is not needed for everything that we will be doing, the examples and the theory are somewhat simplified if we make the following assumption.

\subsubsection{Dimension assumption}

The constraints and the orbit directions span the entire tangent space to the configuration space:

$$
\mathcal{D}_{q}+T_{q} \operatorname{Orb}(q)=T_{q} Q
$$

If this condition is satisfied, we say that the principal case holds.

Let $\mathcal{S}$ be the sub-bundle of $\mathcal{D}$ whose fibre at $q$ is $\mathcal{S}_{q}=\mathcal{D}_{q} \cap T_{q} \operatorname{Orb}(q)$. We assume in this article that $\mathcal{S}_{q} \neq\{0\} .{ }^{5}$ The sub-bundle $\mathcal{S}$ is invariant with respect to the action of $G$ on $T Q$ induced by the left action of $G$ on $Q$. Choose subspaces $\mathcal{U}_{q} \subset \operatorname{Orb}(q)$ such that $\operatorname{Orb}(q)=\mathcal{S}_{q} \oplus \mathcal{U}_{q}$ and the sub-bundle $\mathcal{U}$ is $G$-invariant. Since the distributions $\mathcal{S}$ and $\mathcal{U}$ are left-invariant, there exist subspaces $\mathfrak{b}_{q}^{\mathcal{S}}$ and $\mathfrak{b}_{q}^{\mathcal{U}}$ of the Lie algebra $\mathfrak{g}$ such that in a local trivialization $\mathcal{S}_{q}=L_{g *} \mathrm{~b}_{q}^{\mathcal{S}}$ and $\mathcal{U}_{q}=L_{g *} \mathrm{~b}_{q}^{\mathcal{U}}$.

Let $\mathfrak{b}^{\mathcal{S}}$ and $\mathfrak{b}^{\mathcal{U}}$ be the bundles over $Q / G$ whose fibres are the subspaces $\mathfrak{b}_{q}^{\mathcal{S}}$ and $\mathfrak{b}_{q}^{\mathcal{U}}$ of the Lie algebra $\mathfrak{g}$. Given $\xi \in \mathfrak{g}$, we write its components along these subspaces as $\xi^{\mathcal{S}}$ and $\xi^{\mathcal{U}}$. Let $\mathcal{A}_{s}$ be a connection defined in a local trivialization by the formula

$$
\mathcal{A}_{s}=\operatorname{Ad}_{g}(\xi+\mathcal{A} \dot{r}),
$$

where $\xi \in \mathfrak{g}$ and where $\mathcal{A}$ is a $\mathfrak{g}$-valued form on $Q / G$. This form is such that the constraints in a local trivialization read

$$
\xi^{\mathcal{U}}+\mathcal{A}^{\mathcal{U}} \dot{r}=0 .
$$

That is, the $\mathcal{U}$-component of the form $\mathcal{A}$ is defined by the constraints. The $\mathcal{S}$-component of $\mathcal{A}$ is arbitrary at the moment; later on we will see how the structure of the Lagrangian affects the choice of this component. 
Let $r=\left(r^{1}, \ldots, r^{\sigma}\right)$ be local coordinates in the shape space $Q / G$, and let $e_{1}(r), \ldots, e_{k}(r)$ be a basis of $\mathfrak{g}=T_{e} G$ for each $r \in Q / G$ such that $e_{1}(r), \ldots, e_{m}(r)$ span $\mathfrak{b}^{\mathcal{S}}$ and $e_{m+1}(r), \ldots, e_{k}(r) \operatorname{span} \mathfrak{b}^{\mathcal{U}}$.

As before, we define the vector fields $u_{1}(q), \ldots, u_{n}(q)$ by formulae (2.12). Thus, the fields $u_{\alpha}, \alpha=1, \ldots, \sigma$, span the horizontal space, the fields $u_{\sigma+a}, a=1, \ldots, m$, span the fibres of $\mathcal{S}$, and the remaining fields span the fibres of $\mathcal{U}$. The components of the velocity vector $\dot{q}$ relative to these vector fields are $\dot{r}$ and $\Omega=\xi+\mathcal{A} \dot{r}$. The constraints in this representation are $\Omega^{\mathcal{U}}=0$.

Using the non-holonomic Hamel Equations (3.4) and the $G$-invariance of the constraint distribution $\mathcal{D}$, one obtains the reduced non-holonomic equations of motion from (2.13) and (2.14) by projecting Equation (2.14) onto the fibres of the bundle $\mathfrak{b}_{\mathcal{S}}^{*}$, and imposing constraints, i.e. setting $\Omega=\Omega^{\mathcal{S}}$.

Summarizing, we have the following theorem.

Theorem 4.1: $\quad$ The reduced non-holonomic dynamics is given by the equations

$$
\begin{gathered}
\frac{\mathrm{d}}{\mathrm{d} t} \frac{\partial l}{\partial \dot{r}}-\frac{\partial l}{\partial r}=-\left\langle\frac{\partial l}{\partial \Omega}, \mathbf{i}_{i} \mathcal{B}+\left\langle\mathbf{i}_{\dot{r}} \gamma, \mathcal{A}\right\rangle-\left\langle\gamma, \mathbf{i}_{i} \mathcal{A}\right\rangle+\left\langle\mathcal{E}, \Omega^{\mathcal{S}}\right\rangle\right\rangle \\
{\left[\frac{\mathrm{d}}{\mathrm{d} t} \frac{\partial l}{\partial \Omega}\right]_{\mathcal{S}}=\left[\operatorname{ad}_{\Omega^{\mathcal{S}}}^{*} \frac{\partial l}{\partial \Omega}+\left\langle\frac{\partial l}{\partial \Omega}, \mathbf{i}_{i} \mathcal{E}\right\rangle\right]_{\mathcal{S}}}
\end{gathered}
$$

In the above, the Lagrangian $l$ is written as a function of $(r, \dot{r}, \Omega), \mathcal{B}$ is the curvature of $\mathcal{A}$ (see (2.17)), and the quantities $\gamma$ and $\mathcal{E}$ are defined by Equations (2.16) and (2.18), respectively. Note that the partial derivatives of $l$ in (4.2) and (4.3) are computed before setting $\Omega=\Omega^{\mathcal{S}}$.

Equations (4.2) and (4.3) can be rewritten as

$$
\begin{aligned}
\frac{\mathrm{d}}{\mathrm{d} t} \frac{\partial l_{c}}{\partial \dot{r}}-\frac{\partial l_{c}}{\partial r} & =-\left\langle\frac{\partial l}{\partial \Omega}, \mathbf{i}_{i} \mathcal{B}+\left\langle\mathbf{i}_{r} \gamma, \mathcal{A}\right\rangle-\left\langle\gamma, \mathbf{i}_{r} \mathcal{A}\right\rangle+\left\langle\mathcal{E}, \Omega^{\mathcal{S}}\right\rangle\right\rangle, \\
\frac{\mathrm{d}}{\mathrm{d} t} \frac{\partial l_{c}}{\partial \Omega^{\mathcal{S}}} & =\left[\operatorname{ad}_{\Omega^{\mathcal{S}}}^{*} \frac{\partial l}{\partial \Omega}+\left\langle\frac{\partial l}{\partial \Omega}, \mathbf{i}_{i} \mathcal{E}\right\rangle\right]_{\mathcal{S}}
\end{aligned}
$$

where $l_{c}\left(r, \dot{r}, \Omega^{\mathcal{S}}\right):=l\left(r, \dot{r}, \Omega^{\mathcal{S}}\right)$ is the constrained reduced Lagrangian. These equations follow directly from (4.2) and (4.3) because

$$
\frac{\partial l_{c}}{\partial \dot{r}}=\left.\frac{\partial l}{\partial \dot{r}}\right|_{\Omega=\Omega^{\mathcal{S}}}, \quad \frac{\mathrm{d}}{\mathrm{d} t} \frac{\partial l_{c}}{\partial \dot{r}}=\left.\frac{\mathrm{d}}{\mathrm{d} t} \frac{\partial l}{\partial \dot{r}}\right|_{\Omega=\Omega^{\mathcal{S}}}, \quad \frac{\partial l_{c}}{\partial r}=\left.\frac{\partial l}{\partial r}\right|_{\Omega=\Omega^{\mathcal{S}}}, \quad \frac{\partial l_{c}}{\partial \Omega^{\mathcal{S}}}=\left.\frac{\partial l}{\partial \Omega^{\mathcal{S}}}\right|_{\Omega=\Omega^{\mathcal{S}}} .
$$

Note that in general

$$
\frac{\partial l_{c}}{\partial \Omega^{\mathcal{S}}} \neq\left.\frac{\partial l}{\partial \Omega}\right|_{\Omega=\Omega^{\mathcal{S}}}
$$

\subsection{The moving body frame and non-holonomic connection}

In the rest of the article, we assume that the Lagrangian equals the kinetic minus potential energy and that the kinetic energy is given by a Riemannian metric on the configuration space $Q$. 
Definition 4.2: Under the dimension assumption and the assumption that the Lagrangian is of the form kinetic minus potential energies, the non-holonomic connection $\mathcal{A}^{\text {nhc }}$ is, by definition, the connection on the principal bundle $Q \rightarrow Q / G$ whose horizontal space at $q \in Q$ is given by the orthogonal complement to the space $\mathcal{S}_{q}=\mathcal{D}_{q} \cap T_{q} \operatorname{Orb}(q)$ within the space $\mathcal{D}_{q}$.

Under the assumption that the distribution $\mathcal{D}$ is invariant and from the fact that the group action preserves orthogonality (since it is assumed to preserve the Lagrangian and hence the kinetic energy metric), it follows that the distribution and the horizontal spaces transform to themselves under the group action. Therefore, the non-holonomic connection in a local trivialization is defined by the formula

$$
\mathcal{A}^{\mathrm{nhc}}=\operatorname{Ad}_{g}(\xi+\mathcal{A} \dot{r})
$$

where $\xi \in \mathfrak{g}$ and where $\mathcal{A}$ is a $\mathfrak{g}$-valued one-form on $Q / G$. Given $\dot{q}=(\dot{r}, \xi) \in T_{q} Q$, the vertical and horizontal components of $\dot{q}$ in a local trivialization are

$$
(0, \xi+\mathcal{A} \dot{r}) \text { and }(\dot{r},-\mathcal{A} \dot{r}) .
$$

Using Definition 4.2, we rewrite $\xi+\mathcal{A} \dot{r}$ in (4.4) as

$$
\left(\xi^{\mathcal{S}}+\mathcal{A}^{\mathcal{S}} \dot{r}\right)+\left(\xi^{\mathcal{U}}+\mathcal{A}^{\mathcal{U}} \dot{r}\right), \quad \xi^{\mathcal{S}}+\mathcal{A}^{\mathcal{S}} \dot{r} \in \mathfrak{b}^{\mathcal{S}}, \quad \xi^{\mathcal{U}}+\mathcal{A}^{\mathcal{U}} \dot{r} \in \mathfrak{b}^{\mathcal{U}}
$$

Using the non-holonomic connection, define the body angular velocity $\Omega \in \mathfrak{g}$ by the formula

$$
\Omega=\xi+\mathcal{A} \dot{r}
$$

If the body angular velocity is used, the constraints (4.1) read

$$
\Omega^{\mathcal{U}}=0 .
$$

Let the kinetic energy metric in a local trivialization be written as

$$
\langle\langle\dot{q}, \dot{q}\rangle\rangle=\langle G(r) \dot{r}, \dot{r}\rangle+2\langle\mathcal{K}(r) \dot{r}, \xi\rangle+\langle\mathcal{I}(r) \xi, \xi\rangle .
$$

The constrained locked inertia tensor $\mathcal{I}_{\mathcal{S}}: \mathfrak{b}^{\mathcal{S}} \rightarrow\left(\mathfrak{b}^{\mathcal{S}}\right)^{*}$ is given in a local trivialization by

$$
\left\langle\mathcal{I}_{\mathcal{S}}(r) \xi, \eta\right\rangle=\left\langle\left\langle L_{g *} \xi, L_{g *} \eta\right\rangle\right\rangle, \quad \xi, \eta \in \mathfrak{b}^{\mathcal{S}} .
$$

Similarly, define $\mathcal{I}_{\mathcal{U}}(r): \mathfrak{b}^{\mathcal{U}} \rightarrow\left(\mathfrak{b}^{\mathcal{U}}\right)^{*}, \mathcal{I}_{\mathcal{S U}}(r): \mathfrak{b}^{\mathcal{U}} \rightarrow\left(\mathfrak{b}^{\mathcal{S}}\right)^{*}$, and $\mathcal{I}_{\mathcal{U S}}(r): \mathfrak{b}^{\mathcal{S}} \rightarrow\left(\mathfrak{b}^{\mathcal{U}}\right)^{*}$ by

$$
\begin{array}{rlrl}
\left\langle\mathcal{I}_{\mathcal{U}}(r) \xi, \eta\right\rangle & =\left\langle\left\langle L_{g *} \xi, L_{g *} \eta\right\rangle\right\rangle, & \xi, \eta \in \mathfrak{b}^{\mathcal{U}}, \\
\left\langle\mathcal{I}_{\mathcal{S U}}(r) \xi, \eta\right\rangle & =\left\langle\left\langle L_{g *} \xi, L_{g_{*}} \eta\right\rangle\right\rangle, & & \xi \in \mathfrak{b}^{\mathcal{U}}, \eta \in \mathfrak{b}^{\mathcal{S}}, \\
\left\langle\mathcal{I}_{\mathcal{U S}}(r) \xi, \eta\right\rangle & =\left\langle\left\langle L_{g *} \xi, L_{g *} \eta\right\rangle\right\rangle, & & \xi \in \mathfrak{b}^{\mathcal{S}}, \eta \in \mathfrak{b}^{\mathcal{U}},
\end{array}
$$

respectively.

Definition 4.2 implies that the constrained kinetic energy metric written as a function of $\left(\dot{r}, \Omega^{\mathcal{S}}\right)$ is block-diagonal, that is, it reads

$$
\left\langle G_{c}(r) \dot{r}, \dot{r}\right\rangle+\left\langle\mathcal{I}_{\mathcal{S}}(r) \Omega^{\mathcal{S}}, \Omega^{\mathcal{S}}\right\rangle
$$


Substituting $\xi=\Omega^{\mathcal{S}}-\mathcal{A} \dot{r}$ in (4.5), one obtains

$$
\left\langle G_{c}(r) \dot{r}, \dot{r}\right\rangle+2\left\langle\mathcal{K}_{\mathcal{S}}(r) \dot{r}, \Omega^{\mathcal{S}}\right\rangle+\left\langle\mathcal{I}_{\mathcal{S}}(r) \Omega^{\mathcal{S}}, \Omega^{\mathcal{S}}\right\rangle-2\left\langle\mathcal{I}_{\mathcal{S}}(r) \mathcal{A}^{\mathcal{S}} \dot{r}, \Omega^{\mathcal{S}}\right\rangle-2\left\langle\mathcal{I}_{\mathcal{S U}}(r) \mathcal{A}^{\mathcal{U}} \dot{r}, \Omega^{\mathcal{S}}\right\rangle
$$

and therefore

$$
\mathcal{A}^{\mathcal{S}}=\mathcal{I}_{\mathcal{S}}^{-1}(r) \mathcal{K}_{\mathcal{S}}(r)-\mathcal{I}_{\mathcal{S}}^{-1}(r) \mathcal{I}_{\mathcal{S U}}(r) \mathcal{A}^{\mathcal{U}}
$$

In the rest of the article, we follow the following index conventions:

(1) The first batch of indices range from 1 to $m$ corresponding to the symmetry directions along the constraint space. These indices will be denoted $a, b, c, \ldots$

(2) The second batch of indices range from $m+1$ to $k$ corresponding to the symmetry directions not aligned with the constraints. Indices for this range will be denoted by $a^{\prime}, b^{\prime}, c^{\prime}, \ldots$

(3) The indices $A, B, C, \ldots$ on the Lie algebra $g$ range from 1 to $k$.

(4) The indices $\alpha, \beta, \ldots$ on the shape variables $r$ range from 1 to $\sigma$. Thus, $\sigma$ is the dimension of the shape space $Q / G$ and so $\sigma=n-k$.

The summation convention for all of these indices will be understood.

In the basis $\partial / \partial r^{1}, \ldots, \partial / \partial r^{\sigma}, e_{1}(r), \ldots, e_{k}(r)$, with $e_{1}(r), \ldots, e_{m}(r)$ spanning $\mathfrak{b}^{\mathcal{S}}$ and $e_{m+1}(r), \ldots e_{k}(r)$ spanning $\mathfrak{b}^{\mathcal{U}}$, the components of the non-holonomic connection are

$$
\mathcal{A}_{\alpha}^{a}=\mathcal{I}_{\mathcal{S}}^{a b} \mathcal{K}_{\alpha b}-\mathcal{I}_{\mathcal{S}}^{a b} \mathcal{I}_{b a^{\prime}} \mathcal{A}_{\alpha}^{a^{\prime}} \text { and } \mathcal{A}_{\alpha}^{a^{\prime} 6}
$$

Note that the $\mathcal{I}_{\mathcal{S}}^{a b}$ need not equal $\mathcal{I}^{a b}$.

One often uses the non-holonomic momentum relative to the body frame $p=\partial l_{c} / \partial \Omega^{\mathcal{S}}$ and writes the reduced dynamics as

$$
\begin{aligned}
\frac{\mathrm{d}}{\mathrm{d} t} \frac{\partial l_{c}}{\partial \dot{r}}-\frac{\partial l_{c}}{\partial r}= & -\left\langle p, \frac{\partial \mathcal{I}_{\mathcal{S}}^{-1}}{\partial r} p\right\rangle \\
& -\left\langle p+\mathcal{I}_{\mathcal{U S}} \mathcal{I}_{\mathcal{S}}^{-1} p+\mathbf{i}_{r} \Lambda, \mathbf{i}_{i} \mathcal{B}+\left\langle\mathbf{i}_{r} \gamma, \mathcal{A}\right\rangle-\left\langle\gamma, \mathbf{i}_{i} \mathcal{A}\right\rangle+\left\langle\mathcal{E}, \mathcal{I}_{\mathcal{S}}^{-1} p\right\rangle\right\rangle, \\
\dot{p}= & {\left[\operatorname{ad}_{\mathcal{I}_{\mathcal{S}}^{-1} p}^{*} p+\left\langle p, \mathbf{i}_{r} \tau\right\rangle+\left\langle\mathbf{i}_{r} \Lambda, \mathbf{i}_{r} \mathcal{E}\right\rangle\right]_{\mathcal{S}} . }
\end{aligned}
$$

Equation (4.7) is called the momentum equation in body representation.

In these equations the reduced Lagrangian is represented as a function of $(r, \dot{r}, p)$, $\Lambda$ is a $\left(\mathfrak{b}^{\mathcal{U}}\right)^{*}$-valued one-form on $Q / G$ given by

$$
\Lambda=\left(\mathcal{K}_{\mathcal{U}}-\mathcal{I}_{\mathcal{U S}} \mathcal{A}^{\mathcal{S}}-\mathcal{I}_{\mathcal{U}} \mathcal{A}^{\mathcal{U}}\right) \mathrm{d} r
$$

and $\tau$ is a $\mathfrak{b}^{\mathcal{S}} \otimes\left(\mathfrak{b}^{\mathcal{S}}\right)^{*}$-valued one-forms on $Q / G$ defined by the formula

$$
\left\langle p, \mathbf{i}_{i} \tau\right\rangle=\left[\operatorname{ad}_{\mathcal{I}_{\mathcal{S}}^{-1} p}^{*} \mathbf{i}_{i} \cdot \Lambda+\left\langle p+\mathcal{I}_{\mathcal{U S}} \mathcal{I}_{\mathcal{S}}^{-1} p, \mathbf{i}_{i} \mathcal{E}\right\rangle\right]_{\mathcal{S}} .
$$

Remark: The first term in the right-hand side of the shape Equation (4.6) appears for the following reason. The term $\frac{1}{2}\left\langle\mathcal{I}_{\mathcal{S}} \Omega^{\mathcal{S}}, \Omega^{\mathcal{S}}\right\rangle$ in the constrained reduced Lagrangian $l_{c}\left(r, \dot{r}, \Omega^{\mathcal{S}}\right)$ produces the term $\frac{1}{2}\left\langle\partial_{r} \mathcal{I}_{\mathcal{S}} \Omega^{\mathcal{S}}, \Omega^{\mathcal{S}}\right\rangle$ in the right-hand side of the shape equation. When $p$ is used instead of $\Omega$, this term becomes $\frac{1}{2}\left\langle\partial_{r} \mathcal{I}_{\mathcal{S}} \mathcal{I}_{\mathcal{S}}^{-1} p, \mathcal{I}_{\mathcal{S}}^{-1} p\right\rangle=\frac{1}{2}\left\langle\mathcal{I}_{\mathcal{S}}^{-1} \partial_{r} \mathcal{I}_{\mathcal{S}} \mathcal{I}_{\mathcal{S}}^{-1} p, p\right\rangle=$ $-\frac{1}{2}\left\langle p, \partial_{r} \mathcal{I}_{\mathcal{S}}^{-1} p\right\rangle$. On the other hand, the contribution of the term $\frac{1}{2}\left\langle p, \mathcal{I}_{\mathcal{S}}^{-1} p\right\rangle$ in $l_{c}(r, \dot{r}, p)$ 
is $\frac{1}{2}\left\langle p, \partial_{r} \mathcal{I}_{\mathcal{S}}^{-1} p\right\rangle$. Thus, in order to obtain the correct term, $-\frac{1}{2}\left\langle p, \partial_{r} \mathcal{I}_{\mathcal{S}}^{-1} p\right\rangle$, one needs to subtract $\left\langle p, \partial_{r} \mathcal{I}_{\mathcal{S}}^{-1} p\right\rangle$ from the right-hand side of the shape equation.

Sometimes it is desirable to use a slightly different representation of Equations (4.2), (4.3) and (4.6), (4.7). Recall that the inertia tensor is an invertible operator $\mathcal{I}: \mathfrak{g} \rightarrow \mathfrak{g}^{*}$. Its inverse $\mathcal{I}^{-1}$ maps $\mathfrak{g}^{*}$ to $\mathfrak{g}$, and defines the operators

$$
\mathcal{I}^{\mathcal{S}}: \mathfrak{b}_{\mathcal{S}}^{*} \rightarrow \mathfrak{b}^{\mathcal{S}}, \quad \mathcal{I}^{\mathcal{U}}: \mathfrak{b}_{\mathcal{U}}^{*} \rightarrow \mathfrak{b}^{\mathcal{U}}, \quad \mathcal{I}^{\mathcal{S U}}: \mathfrak{b}_{\mathcal{U}}^{*} \rightarrow \mathfrak{b}^{\mathcal{S}}, \quad \mathcal{I}^{\mathcal{U S}}: \mathfrak{b}_{\mathcal{S}}^{*} \rightarrow \mathfrak{b}^{\mathcal{U}}
$$

by the formulae

$$
\begin{aligned}
& \mathcal{I}^{-1} \alpha=\mathcal{I}^{\mathcal{S}} \alpha+\mathcal{I}^{\mathcal{U S}} \alpha, \quad \mathcal{I}^{\mathcal{S}} \alpha \in \mathfrak{b}^{\mathcal{S}}, \quad \mathcal{I}^{\mathcal{U S}} \alpha \in \mathfrak{b}^{\mathcal{U}}, \quad \alpha \in \mathfrak{b}_{\mathcal{S}}^{*}, \\
& \mathcal{I}^{-1} \alpha=\mathcal{I}^{\mathcal{S U}} \alpha+\mathcal{I}^{\mathcal{U}} \alpha, \quad \mathcal{I}^{\mathcal{S U}} \alpha \in \mathfrak{b}^{\mathcal{S}}, \quad \mathcal{I}^{\mathcal{U}} \alpha \in \mathfrak{b}^{\mathcal{U}}, \quad \alpha \in \mathfrak{b}_{\mathcal{U}}^{*} .
\end{aligned}
$$

The operators (4.8) are uniquely defined by these formulae since $\mathfrak{g}=\mathfrak{b}^{\mathcal{S}} \oplus \mathfrak{b}^{\mathcal{U}}$. Note that $\mathcal{I}^{\mathcal{D U}}$ and $\mathcal{I}^{\mathcal{U S}}$ normally are not zero operators. However, it is straightforward to see that

$$
\mathcal{I}^{\mathcal{U S}} \mathcal{I}_{\mathcal{S}}+\mathcal{I}^{\mathcal{U}} \mathcal{I}_{\mathcal{U S}}: \mathfrak{b}^{\mathcal{S}} \rightarrow \mathfrak{b}^{\mathcal{U}}
$$

maps every $\xi \in \mathfrak{b}^{\mathcal{S}}$ to 0 . Therefore,

$$
\left(\mathcal{I}^{\mathcal{U}}\right)^{-1}\left(\mathcal{I}^{\mathcal{U S}} \mathcal{I}_{\mathcal{S}}+\mathcal{I}^{\mathcal{U}} \mathcal{I}_{\mathcal{U S}}\right) \mathcal{I}_{\mathcal{S}}^{-1}=\left(\mathcal{I}^{\mathcal{U}}\right)^{-1} \mathcal{I}^{\mathcal{U S}}+\mathcal{I}_{\mathcal{U S}} \mathcal{I}_{\mathcal{S}}^{-1}=0
$$

and one can replace the terms $\mathcal{I}_{\mathcal{U S}} \mathcal{I}_{\mathcal{S}}^{-1} p$ with $-\left(\mathcal{I}^{\mathcal{U}}\right)^{-1} \mathcal{I}^{\mathcal{U S}} p$.

In coordinate notation, the reduced equations of motion become

$$
\begin{aligned}
\frac{\mathrm{d}}{\mathrm{d} t} \frac{\partial l_{c}}{\partial \dot{r}^{\alpha}}-\frac{\partial l_{c}}{\partial r^{\alpha}}= & -\mathcal{D}_{b \alpha}^{c} \mathcal{I}_{\mathcal{S}}^{b d} p_{c} p_{d}-\mathcal{K}_{\alpha \beta \gamma} \dot{r}^{\beta} \dot{r}^{\gamma} \\
& -\left(\mathcal{B}_{\alpha \beta}^{c}-\mathcal{I}_{c^{\prime} a^{\prime}}^{\mathcal{U}} \mathcal{I}^{a^{\prime} c} \mathcal{B}_{\alpha \beta}^{c^{\prime}}+\mathcal{D}_{b \beta \alpha} \mathcal{I}_{\mathcal{S}}^{b c}\right) p_{c} \dot{r}^{\beta} \\
\dot{p}_{a}= & \left(C_{b a}^{c}-C_{b a}^{c^{\prime}} \mathcal{I}_{c^{\prime} a^{\prime}}^{\mathcal{U}} \mathcal{I}^{a^{\prime} c}\right) \mathcal{I}_{\mathcal{S}}^{b d} p_{c} p_{d}+\mathcal{D}_{a \alpha}^{c} p_{c} \dot{r}^{\alpha}+\mathcal{D}_{a \alpha \beta} \dot{r}^{\alpha} \dot{r}^{\beta} .
\end{aligned}
$$

Here and below $l_{c}\left(r^{\alpha}, \dot{r}^{\alpha}, \Omega^{a}\right)$ is the constrained Lagrangian, and $\mathcal{I}_{\mathcal{S}}^{b d}$ and $\mathcal{I}_{a^{\prime} c^{\prime}}^{\mathcal{U}}$ are the components of the tensors $\mathcal{I}_{D}^{-1}$ and $\left(\mathcal{I}^{\mathcal{U}}\right)^{-1}$, respectively. We stress that in general $\mathcal{I}_{\mathcal{S}}^{b d} \neq \mathcal{I}^{b d}$ and $\mathcal{I}_{a^{\prime} c^{\prime}}^{\mathcal{U}} \neq \mathcal{I}_{a^{\prime} c^{\prime}}$. The coefficients $\mathcal{B}_{\alpha \beta}^{C}, \mathcal{D}_{b \alpha}^{c}, \mathcal{D}_{b \alpha \beta}$ and $\mathcal{K}_{\alpha \beta \gamma}$ are given by the formulae

$$
\begin{aligned}
\mathcal{B}_{\alpha \beta}^{C} & =\frac{\partial \mathcal{A}_{\alpha}^{C}}{\partial r^{\beta}}-\frac{\partial \mathcal{A}_{\beta}^{C}}{\partial r^{\alpha}}-C_{B A}^{C} \mathcal{A}_{\alpha}^{A} \mathcal{A}_{\beta}^{B}+\gamma_{A \beta}^{C} \mathcal{A}_{\alpha}^{A}-\gamma_{A \alpha}^{C} \mathcal{A}_{\beta}^{A}, \\
\mathcal{D}_{b \alpha}^{c} & =-\left(C_{A b}^{c}-C_{A b}^{c^{\prime}} \mathcal{I}_{c^{\prime} a^{\prime}}^{\mathcal{U}} \mathcal{I}^{a^{\prime} c}\right) \mathcal{A}_{\alpha}^{A}+C_{a b}^{c^{\prime}} \Lambda_{c^{\prime} \alpha} \mathcal{I}_{\mathcal{S}}^{a c}+\gamma_{b \alpha}^{c}-\gamma_{b \alpha}^{c^{\prime}} \mathcal{I}_{c^{\prime} a^{\prime}}^{\mathcal{U}} \mathcal{I}^{a^{\prime} c}, \\
\mathcal{D}_{b \alpha \beta} & =\Lambda_{c^{\prime} \beta}\left(\gamma_{b \alpha}^{c^{\prime}}-C_{A b}^{c^{\prime}} \mathcal{A}_{\alpha}^{A}\right), \\
\mathcal{K}_{\alpha \beta \gamma} & =\Lambda_{c^{\prime} \gamma} \mathcal{B}_{\alpha \beta}^{c^{\prime}},
\end{aligned}
$$

and $\gamma_{b \alpha}^{C}$ are the components of the form $\gamma$ introduced in (2.16). Equations (4.9) and (4.10) originated in [10] and [19].

The keys to the qualitative behaviour of this system and stability in particular are the terms on the right-hand side of the momentum Equation (4.10). One case of interest is where the matrix $C_{b a}^{c}-C_{b a}^{c^{\prime}} \mathcal{I}_{c^{\prime} a^{\prime}}^{\mathcal{I}} \mathcal{I}^{a^{\prime} c}$ is skew. This is discussed in [12]. This again divides into two cases: the terms quadratic in $\dot{r}$ are present or not. There are cases where these terms vanish and one does not obtain asymptotic stability - for example the 
rolling penny problem. If the terms quadratic in $\dot{r}$ are present one often obtains asymptotic stability, as in the rattleback top.

A key case of interest to us in this article are the Euler-Poincaré-Suslov equations discussed below where there are no internal or shape degrees of freedom, i.e. no coordinates $r^{\alpha}$. We discuss in detail in this case when one does or does not obtain partial asymptotic stability.

Whether the non-holonomic systems exhibit asymptotic behaviour or not it is striking that we have the following proposition.

Proposition 4.3: The non-holonomic equations (4.9) and (4.10), in the case that $l_{c}$ is quadratic in $p$ and $\dot{r}$, are time reversible.

Proof: The equations are verified to be invariant under the discrete $\mathbb{Z}_{2}$ symmetry

$$
(t \rightarrow-t, p \rightarrow-p, \dot{r} \rightarrow-\dot{r}),
$$

giving the stated result.

Furthermore, in this setting it is easy to check that energy is always preserved. This is in contrast to the type of asymptotic stability exhibited by, say a harmonic oscillator with external dissipation (friction), which is not reversible.

\subsection{Momentum conservation relative to the body frame}

Here we discuss how one can select the basis in $\mathcal{D}_{q} \cap T_{q} \operatorname{Orb}(q)$ in order to observe momentum conservation in the non-holonomic setting. More details can be found in [20].

Theorem 4.4: For $\eta(r) \in \mathfrak{b}^{\mathcal{S}}$, the quantity $\langle p, \eta(r)\rangle$ is a conservation law of the reduced non-holonomic dynamics (4.6) and (4.7) if

(i) The form $\tau$ is exact;

(ii) The component of $\left[\operatorname{ad}_{\mathcal{I}_{\mathcal{S}}^{-1} p}^{*} p+\left\langle\mathbf{i}_{i} \Lambda, \mathbf{i}_{i} \mathcal{E}\right\rangle\right]_{\mathcal{S}}$ along $\eta(r)$ equals zero, i.e.,

$$
\left\langle\left[\operatorname{ad}_{\mathcal{I}_{\mathcal{S}}^{-1} p}^{*} p+\left\langle\mathbf{i}_{i} \Lambda, \mathbf{i}_{i} \mathcal{E}\right\rangle\right]_{\mathcal{S}}, \eta(r)\right\rangle=0 .
$$

Proof: Taking the flow derivative of $\langle p, \eta(r)\rangle$, we obtain

$$
\frac{\mathrm{d}}{\mathrm{d} t}\langle p, \eta(r)\rangle=\langle\dot{p}, \eta(r)\rangle+\langle p, \dot{\eta}\rangle=\left\langle\left[\operatorname{ad}_{\mathcal{I}_{\mathcal{S}}^{-1} p}^{*} p+\left\langle p, \mathbf{i}_{i} \tau\right\rangle+\left\langle\mathbf{i}_{r} \Lambda, \mathbf{i}_{i} \mathcal{E}\right\rangle\right]_{\mathcal{S}}, \eta(r)\right\rangle+\langle p, \dot{\eta}\rangle .
$$

Condition (ii) implies

$$
\frac{\mathrm{d}}{\mathrm{d} t}\langle p, \eta(r)\rangle=\left\langle\left[\left\langle p, \mathbf{i}_{i} \tau\right\rangle\right]_{\mathcal{S}}, \eta(r)\right\rangle+\langle p, \dot{\eta}\rangle=\left\langle p,\left\langle\mathbf{i}_{i} \tau, \eta\right\rangle+\dot{\eta}\right\rangle .
$$

Equation

$$
\mathrm{d} \eta=-\langle\tau, \eta\rangle
$$

implies that the flow derivative of $\langle p, \eta(r)\rangle$ vanishes. Condition (i) of the theorem ensures the existence of $\eta(r) \in \mathfrak{b}^{\mathcal{S}}$. 
Corollary 4.5: Assume that the form $\tau$ is exact and $\left[\operatorname{ad}_{\mathcal{I}_{S}^{-1} p}^{*} p+\left\langle\mathbf{i}_{\dot{r}} \Lambda, \mathbf{i}_{\dot{r}} \mathcal{E}\right\rangle\right]_{\mathcal{S}}=0$. Then there exists a basis $\eta_{1}(r), \ldots, \eta_{m}(r)$ of $\mathfrak{b}^{\mathcal{S}}$ such that the momentum equation written relative to this basis becomes

$$
\dot{p}=0 \text {. }
$$

This basis, if it exists, is often non-trivial even for simple (e.g. commutative) symmetry groups.

Example: Consider a system whose Lagrangian $L: T \mathbb{R}^{3} \rightarrow \mathbb{R}$ and constraint are

$$
L=\frac{1}{2}\left(\dot{r}^{2}+\left(1-b^{2}(r)\right)\left(\dot{s}^{1}\right)^{2}+\left(\dot{s}^{2}\right)^{2}\right)-V(r)
$$

and

$$
\dot{s}^{2}=b(r) \dot{s}^{1},
$$

respectively. This system is $\mathbb{R}^{2}$-invariant. The group elements are written as $s=\left(s^{1}, s^{2}\right) \in \mathbb{R}^{2}$. The potential energy $V(r)$ and the constraint coefficient $b(r)$ are smooth functions of the shape variable $r$. The subspace $\mathfrak{b}^{\mathcal{S}}$ of the Lie algebra $\mathbb{R}^{2}$ for this system is one-dimensional; it is spanned by

$$
\frac{\partial}{\partial s^{1}}+b(r) \frac{\partial}{\partial s^{2}} .
$$

The momentum equation for this system is computed to be

$$
\dot{p}=b(r) b^{\prime}(r) p \dot{r} .
$$

The right-hand side of the momentum equation satisfies the conditions of Corollary 4.5 .

Using (4.12), $\mathrm{d} \eta=-b(r) b^{\prime}(r) \eta \mathrm{d} r$, which implies

$$
\eta(r)=e^{-\frac{1}{2} b^{2}(r)}\left(\partial_{s^{1}}+b(r) \partial_{s^{2}}\right) .
$$

Thus, if one defines the vector fields $u_{1}, u_{2}, u_{3} \in T \mathbb{R}^{3}$ by the formulae

$$
u_{1}=\frac{\partial}{\partial r}, \quad u_{2}=e^{-\frac{1}{2} b^{2}(r)}\left(\frac{\partial}{\partial s^{1}}+b(r) \frac{\partial}{\partial s^{2}}\right), \quad u_{3}=\frac{\partial}{\partial s^{2}},
$$

non-holonomic momentum conservation is observed.

\section{Euler-Poincaré-Suslov equations}

An important special case of the reduced non-holonomic equations is the following: The configuration space is a Lie group $G$ and the system is characterized by a left-invariant Lagrangian $l=\frac{1}{2}\langle\mathcal{I} \Omega, \Omega\rangle=\frac{1}{2} \mathcal{I}_{A B} \Omega^{A} \Omega^{B}$, where $\Omega=g^{-1} \dot{g} \in \mathfrak{g}, \mathcal{I}_{A B}$ are the components of the inertia tensor $\mathcal{I}: \mathfrak{g} \mapsto \mathfrak{g}^{*}$, and by the left-invariant constraint

$$
\langle a, \Omega\rangle=a_{A} \Omega^{A}=0,
$$

where $a$ lies in the dual space $\mathfrak{g}^{*}$ and $\langle\cdot, \cdot\rangle$ denotes the natural pairing between the Lie algebra and its dual. Multiple constraints may be imposed as well. 
In the absence of constraints the reduced dynamics is governed by the Euler-Poincare Equations (2.23). Here we represent these equations as

$$
\dot{p}=\operatorname{ad}_{\Omega}^{*} p
$$

where $p=\mathcal{I} \Omega \in \mathfrak{g}^{*}$ is the body momentum. In components, these equations read

$$
\dot{p}_{B}=C_{A B}^{C} \mathcal{I}^{A D} p_{C} p_{D}=C_{A B}^{C} p_{C} \Omega^{A} .
$$

\subsection{Measure Preservation}

It is of interest that while the unconstrained dynamics on $T G$ preserves the phase volume, the reduced dynamics (5.2) may fail to be measure-preserving and thus may exhibit asymptotic behaviour, as was shown by [21]. See [34-39] for the use of invariant measure in understanding dynamics and integrability of non-Hamiltonian mechanics.

Theorem 5.1: The Euler-Poincaré Equations (5.2) have an integral invariant with positive $C^{1}$ density if and only if the group $G$ is unimodular. ${ }^{7}$

We only outline the proof here, for the complete exposition refer to [21]. Sufficiency can be seen as follows: A criterion for unimodularity ([21]) is $C_{A C}^{C}=0$ (with the summation convention). Since the divergence of the right hand side of Equation (5.3) is $C_{A C}^{C} \mathcal{I}^{A D} p_{D}=0$, the corresponding flow preserves the volume form $d p_{1} \wedge \cdots \wedge d p_{k}$ in $\mathfrak{g}^{*}$. Recall that the flow of a vector field $f$ is volume-preserving if and only if $\operatorname{div} f=0$. The necessity is derived from the following theorem of [21]: A flow of a homogeneous vector field in $\mathbb{R}^{n}$ has an integral invariant with positive $C^{1}$ density if and only if this flow preserves the standard volume in $\mathbb{R}^{n}$.

Now, turning to the case where we have the constraint (5.1) we obtain the EulerPoincaré-Suslov equations

$$
\dot{p}=\operatorname{ad}_{\Omega}^{*} p+\lambda a
$$

together with the constraint (5.1), where $\lambda$ is the Lagrange multiplier. Using (3.8), we obtain

$$
\lambda=-\left\langle\operatorname{ad}_{\Omega}^{*} p, \mathcal{I}^{-1} a\right\rangle /\left\langle a, \mathcal{I}^{-1} a\right\rangle=-\left\langle p, \operatorname{ad}_{\Omega} \mathcal{I}^{-1} a\right\rangle /\left\langle a, \mathcal{I}^{-1} a\right\rangle .
$$

One can then formulate a condition for the existence of an invariant measure of the Euler-Poincaré-Suslov equations. The following result was proved by [21] for compact algebras and for arbitrary algebras by [22].

Theorem 5.2: The Euler-Poincaré-Suslov Equations (5.4) have an integral invariant with positive $C^{1}$ density if and only if

$$
\operatorname{Kad}_{\mathcal{I}^{-1} a}^{*} a+T=\gamma a
$$

for some $\gamma \in \mathbb{R}$, where $K=1 /\left\langle a, \mathcal{I}^{-1} a\right\rangle$ and $T \in \mathfrak{g}^{*}$ is defined by $\langle T, \xi\rangle=\operatorname{Tr}\left(\operatorname{ad}_{\xi}\right)$.

In coordinates condition (5.5) reads

$$
K C_{A B}^{C} \mathcal{I}^{A D} a_{C} a_{D}+C_{B C}^{C}=\gamma a_{B} .
$$

The proof is done by solving for the Lagrange multiplier $\lambda$ and computing the divergence of the right-hand side.

For a unimodular Lie group the quantities $\sum_{B=1}^{k} C_{A B}^{B}, A=1, \ldots, k$, always vanish. In particular if the group is compact or semisimple, then the group is unimodular. 
Further, in the semisimple case we can use the Killing form to identify $\mathfrak{g}^{*}$ with $\mathfrak{g}$ and condition (5.6) can be written as

$$
\left[\square^{-1} a, a\right]=\gamma a, \quad \gamma \in \mathbb{R} .
$$

For a matrix Lie algebra the Killing form is a multiple of the trace and so pairing $a$ with itself (via the Killing form or multiple of the trace) we have

$$
\left\langle\left[\mathcal{I}^{-1} a, a\right], a\right\rangle=\langle\gamma a, a\rangle
$$

and, since the left-hand side is zero, we conclude that $\gamma$ must be zero. Thus in this case only constraint vectors $a$ which commute with $\mathcal{I}^{-1} a$ allow volume in $\mathfrak{g}^{*}$ to be preserved.

This means that $a$ and $\mathcal{I}^{-1} a$ must lie in the same maximal commuting subalgebra. In particular if $a$ is an eigenstate of the inertia tensor, the flow is measure-preserving. In the case that the maximal commuting subalgebra is one-dimensional this is a necessary condition. This is the case for groups such as $\mathrm{SO}(3)$ (see below). One can introduce several constraints of this type.

We can thus formulate the following result as a symmetry requirement on the constraints.

Theorem 5.3: A compact Euler-Poincaré-Suslov system is volume-preserving (and therefore generically cannot exhibit asymptotic dynamics in the momentum space) if the constraint vectors a are eigenvectors of the inertia tensor, or the constrained system is $\mathbb{Z}_{2}$ symmetric about all principal axes. If the maximal commuting subalgebra is one-dimensional this condition is necessary.

In the case when the group is not compact a little more freedom is allowed as we shall see in the case of the Chaplygin sleigh below.

\subsection{Spatial momentum dynamics}

Let $e_{A}$ be a basis of $\mathfrak{g}$, and let $u_{A}=R_{g} * e_{A}$. Then $\dot{g}=\langle u(g), \eta\rangle$, where $\eta=\dot{g} g^{-1}$. If $G=\operatorname{SO}(3)$, $\eta$ is the spatial angular velocity.

The Lagrangian and the constraints are

$$
l^{R}(g, \eta)=\frac{1}{2}\langle\square(g) \eta, \eta\rangle
$$

and

$$
\left\langle a^{R}(g), \eta\right\rangle=0,
$$

respectively. In the above, $\mathbb{\square}(g)=\operatorname{Ad}_{g^{-1}}^{*} \mathcal{I} \operatorname{Ad}_{g^{-1}}$ and $a^{R}(g)=\operatorname{Ad}_{g^{-1}}^{*} a \in \mathfrak{g}^{*}$.

Define the spatial momentum $\mu$ by the formula

$$
\mu=\frac{\partial l^{R}}{\partial \eta}=\operatorname{Ad}_{g^{-1}}^{*} p .
$$

Then, using (3.6), we obtain

$$
\dot{\mu}=\lambda a^{R}(g),
$$


where, according to (3.8)

$$
\lambda=-\left\langle\mu, \dot{b}^{R}(g)\right\rangle \cdot\left\langle a^{R}(g), b^{R}(g)\right\rangle^{-1} .
$$

Equation (5.7) implies that the spatial momentum is not generically conserved in the non-holonomic setting.

\section{Examples}

In this section, we illustrate the above theory by discussing a number of key nonholonomic examples where the dynamics is conveniently described using quasivelocities. Perhaps the simplest non-holonomic system is the following:

\subsection{Euler-Poincaré-Suslov problem on $\mathrm{SO}(3)$}

In this case, one can formulate the problem as the standard Euler equations

$$
\mathcal{I} \dot{\Omega}=\mathcal{I} \Omega \times \Omega,
$$

where $\Omega=\left(\Omega_{1}, \Omega_{2}, \Omega_{3}\right)$ is the system angular velocities in a frame where the inertia matrix is of the form $\mathcal{I}=\operatorname{diag}\left(I_{1}, I_{2}, I_{3}\right)$ and the system is subject to the constraint

$$
a \cdot \Omega=0,
$$

where $a=\left(a_{1}, a_{2}, a_{3}\right)$.

The non-holonomic equations of motion are then given by

$$
\mathcal{I} \dot{\Omega}=\mathcal{I} \Omega \times \Omega+\lambda a, \quad a \cdot \Omega=0,
$$

and the Lagrange multiplier is given by

$$
\lambda=-\frac{\mathcal{I}^{-1} a \cdot(\mathcal{I} \Omega \times \Omega)}{\mathcal{I}^{-1} a \cdot a} .
$$

One can then check that if $a_{2}=a_{3}=0$ (a constraint that is an eigenstate of the moment of inertia operator) for example, the (reduced) phase volume is preserved.

\subsection{Chaplygin sleigh}

Here we describe the Chaplygin sleigh, one of the simplest mechanical system which illustrates the possible dissipative nature of non-holonomic systems. Note that this dissipation is 'non-physical' in the sense that the energy is preserved while the volume on the level surfaces of energy is not.

The Chaplygin sleigh is discussed for example in [1] (see also the paper [13], where an interesting connection with systems with impacts is made).

The sleigh is essentially a flat rigid body in the plane supported at three points, two of which slide freely without friction while the third is a knife edge constraint which allows no motion perpendicular to its edge.

We will derive the equations from the Euler-Poincaré-Suslov equations (5.4) on SE(2). Let $\theta$ be the angular orientation of the sleigh, $(x, y)$ be the coordinates of the contact point 
as shown in Figure 1. The blade is depicted as a bold segment in the Figure. The body frame is

$$
e_{1}=\frac{\partial}{\partial \theta}, \quad e_{2}=\cos \theta \frac{\partial}{\partial x}+\sin \theta \frac{\partial}{\partial y}, \quad e_{3}=-\sin \theta \frac{\partial}{\partial x}+\cos \theta \frac{\partial}{\partial y}
$$

(Figure 1); the vector $e_{1}$ may be visualized as a vector orthogonal to the body and directed towards the reader. Let $\Omega=g^{-1} \dot{g} \in \mathfrak{s e}(2)$, the components of $\Omega$ relative to the body frame are $\left(\Omega^{1}, \Omega^{2}, \Omega^{3}\right)$, where $\Omega^{1}=\dot{\theta}$ is the angular velocity of the sleigh relative to the vertical line through the contact point, and $\Omega^{2}$ and $\Omega^{3}$ are the components of linear velocity of the contact point in the directions along and orthogonal to the blade, respectively. Let the centre of mass be located on the line through the blade at the point $a e_{2}$ relative to the body frame. See Figure 1 for details. Denote the mass and the moment of inertia of the sleigh by $m$ and $J$. The constraint reads $\Omega^{3}=0$.

The velocity of the centre of mass relative to the body frame is $\Omega^{2} e_{2}+\left(\Omega^{3}+a \Omega^{1}\right) e_{3}$. The Lagrangian is just the kinetic energy of the sleigh, which is the sum of the kinetic energies of the linear and rotational modes of the body. Therefore the reduced Lagrangian and the reduced constrained Lagrangian of the Chaplygin sleigh are ([16])

$$
l(\Omega)=\frac{1}{2}\left(\left(J+m a^{2}\right)\left(\Omega^{1}\right)^{2}+m\left(\left(\Omega^{2}\right)^{2}+\left(\Omega^{3}\right)^{2}+2 a \Omega^{1} \Omega^{3}\right),\right.
$$

and

$$
l_{c}(\Omega)=\frac{1}{2}\left(\left(J+m a^{2}\right)\left(\Omega^{1}\right)^{2}+m\left(\Omega^{2}\right)^{2}\right),
$$

respectively. The constrained space $\mathfrak{b}^{\mathcal{S}}$ is defined by

$$
\mathfrak{b}^{\mathcal{S}}=\left\{\Omega^{3}=0\right\} .
$$

The dual of $b^{\mathcal{S}}$ is

$$
\left(b^{\mathcal{S}}\right)^{*}=\left\{p_{3}=0\right\} .
$$

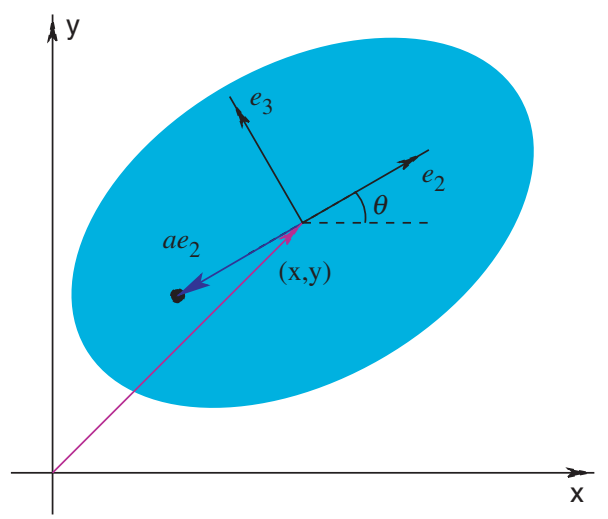

Figure 1. The Chaplygin sleigh. 


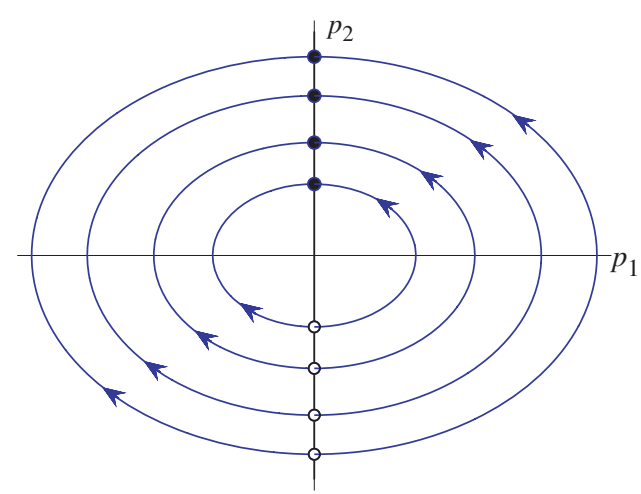

Figure 2. The momentum dynamics of the unbalanced Chaplygin sleigh.

\subsubsection{Equations of motion in the body frame}

The reduced dynamics of the Chaplygin sleigh is given by the Euler-Poincare-Suslov equations on the algebra se(2). The two independent equations are

$$
\dot{p}_{1}=-\frac{a p_{1} p_{2}}{J+m a^{2}}, \quad \dot{p}_{2}=\frac{m a p_{1}^{2}}{\left(J+m a^{2}\right)^{2}},
$$

where

$$
p_{1}=\frac{\partial l_{c}}{\partial \Omega^{1}}=\left(J+m a^{2}\right) \Omega^{1}, \quad p_{2}=\frac{\partial l_{c}}{\partial \Omega^{2}}=m \Omega^{2}
$$

are the components of non-holonomic momentum relative to the body frame. If $a \neq 0$, equations in (6.1) have a family of relative equilibria given by $p_{1}=0, p_{2}=$ const. Linearizing at any of these equilibria, we find that there is one zero eigenvalue and one real eigenvalue. The trajectories of (6.1) are either equilibria situated on the line $p_{1}=0$, or elliptic arcs, as shown in Figure 2. Assuming $a>0$, the equilibria located in the upper half plane are asymptotically stable (filled dots in Figure 2) whereas the equilibria in the lower half plane are unstable (empty dots). The elliptic arcs form heteroclinic connections between the pairs of equilibria.

If $a=0$, momentum dynamics is trivial. Taken together with the analysis of equations in (6.1), this means that we do not observe asymptotic stability if and only if $a=0$.

The condition $a=0$ means that the centre of mass is situated at the contact point of the sleigh and the plane. The inertia tensor $\mathcal{I}$ becomes block-diagonal in this case, i.e. the direction defined by the constraint is an eigendirection of the inertia tensor.

\subsubsection{The dynamics of the spatial momentum}

Recall that the spatial angular velocity is defined as $\eta=R_{g^{-1}}^{*} \dot{g}=\dot{g} g^{-1} \in \mathfrak{s e}(2)$. For the group $\mathrm{SE}(2)$ the components of $\eta$ are computed to be

$$
\eta^{1}=\dot{\theta}, \quad \eta^{2}=\dot{x}+y \dot{\theta}, \quad \eta^{3}=\dot{y}-x \dot{\theta} .
$$


The corresponding frame consists of the generators of the left action of $\operatorname{SE}(2)$ on itself.

The latter are given by the formulae

$$
u_{1}=\frac{\partial}{\partial \theta}-y \frac{\partial}{\partial x}+x \frac{\partial}{\partial y}, \quad u_{2}=\frac{\partial}{\partial x}, \quad u_{3}=\frac{\partial}{\partial y},
$$

and the corresponding brackets are computed to be

$$
\left[u_{1}, u_{2}\right]=-u_{3}, \quad\left[u_{1}, u_{3}\right]=u_{2}, \quad\left[u_{2}, u_{3}\right]=0 .
$$

The Lagrangian becomes

$$
\begin{aligned}
l^{R}(g, \theta)= & \frac{J+m a^{2}}{2}\left(\eta^{1}\right)^{2}+\frac{m}{2}\left(\left(\eta^{2}-y \eta^{1}\right)^{2}+\left(\eta^{3}+x \eta^{1}\right)^{2}\right. \\
& \left.+2 a \eta^{1}\left[-\left(\eta^{2}-y \eta^{1}\right) \sin \theta+\left(\eta^{3}+x \eta^{1}\right) \cos \theta\right]\right),
\end{aligned}
$$

and the constraint becomes

$$
-\left(\eta^{2}-y \eta^{1}\right) \sin \theta+\left(\eta^{3}+x \eta^{1}\right) \cos \theta=0 .
$$

The spatial components of the non-holonomic momentum are

$$
\begin{aligned}
& \mu_{1}=\left(J+m a^{2}\right) \eta^{1}-m y\left(\eta^{2}-y \eta^{1}\right)+m x\left(\eta^{3}+x \eta^{1}\right)+m a \eta^{1}(x \cos \theta+y \sin \theta), \\
& \mu_{2}=m\left[\left(\eta^{2}-y \eta^{1}\right)-a \eta^{1} \sin \theta\right] \\
& \mu_{3}=m\left[\left(\eta^{2}-y \eta^{1}\right)+a \eta^{1} \cos \theta\right] .
\end{aligned}
$$

The momentum belongs to the subspace of $\mathfrak{s e}^{*}(2)$ defined by the equation

$$
-\mu_{2} \sin \theta+\mu_{3} \cos \theta-\frac{m a}{J+m a^{2}}\left(\mu_{1}+y \mu_{2}-x \mu_{3}\right)=0 .
$$

Using (5.7), the dynamics of spatial momentum for the Chaplygin sleigh is

$$
\dot{\mu}_{1}=\lambda(x \cos \theta+y \sin \theta), \quad \dot{\mu}_{2}=-\lambda \sin \theta, \quad \dot{\mu}_{3}=\lambda \cos \theta,
$$

where $\lambda$ is as given by the formula

$$
\lambda=\frac{J\left(\mu_{1}+y \mu_{2}-x \mu_{3}\right)\left(\mu_{2} \cos \theta+\mu_{3} \sin \theta\right)}{\left(J+m a^{2}\right)^{2}} .
$$

Regardless of the value of $a$, the spatial momentum of the Chaplygin sleigh is conserved if and only if $\theta=$ const, i.e. when the contact point of the sleigh is moving along a straight line.

\subsubsection{Momentum conservation for the unbalanced Chaplygin sleigh}

We now show that the components of momentum are conserved if one uses the vector fields $u_{1}, u_{2}, u_{3} \in T \mathrm{SE}(2)$ given by

$$
u_{1}=\cos k \theta \frac{\partial}{\partial \theta}+\frac{a}{k} \sin k \theta\left(\cos \theta \frac{\partial}{\partial x}+\sin \theta \frac{\partial}{\partial y}\right),
$$




$$
\begin{gathered}
u_{2}=-\frac{k}{a} \sin k \theta \frac{\partial}{\partial \theta}+\cos k \theta\left(\cos \theta \frac{\partial}{\partial x}+\sin \theta \frac{\partial}{\partial y}\right) \\
u_{3}=-\sin \theta \frac{\partial}{\partial x}+\cos \theta \frac{\partial}{\partial y}
\end{gathered}
$$

where $k^{2}:=m a^{2} /\left(J+m a^{2}\right)$.

The structure of the momentum trajectories in Figure 2 in combination with translational symmetry of the sleigh suggests that there may exist vector fields $u=u^{1}(\theta) e_{1}+u^{2}(\theta) e_{2}$ such that $\langle p, u\rangle=$ const. That is, the components of the nonholonomic momentum along a field whose direction and magnitude relative to the body frame depends on the angle $\theta$ may be conserved. Note that $\theta$ is not a shape variable as in Theorem 4.4.

Differentiating the quantity $\langle p, u(\theta)\rangle$ and taking into account Equations (6.1) yields

$$
\dot{u}^{1}=-\frac{m a p_{1}}{\left(J+m a^{2}\right)^{2}} u^{2}, \quad \dot{u}^{2}=\frac{a p_{1}}{J+m a^{2}} u^{1} .
$$

Using the formula $p_{1}=\left(J+m a^{2}\right) \Omega^{1}=\left(J+m a^{2}\right) \dot{\theta}$, we obtain

$$
\frac{\mathrm{d} u^{1}}{\mathrm{~d} \theta}=-\frac{m a}{J+m a^{2}} u^{2}, \quad \frac{\mathrm{d} u^{2}}{\mathrm{~d} \theta}=a u^{1} .
$$

This system defines two independent vector fields (6.2) and (6.3). The field $u_{3}$ is defined by formula (6.4) in order to have the sleigh constraint to be written as $\xi^{3}=0$, where $\left(\xi^{1}, \xi^{2}, \xi^{3}\right)$ are the components of $\dot{g}$ relative to the frame $u_{1}, u_{2}, u_{3}$.

Conservation of momentum can be confirmed by the Hamel equations for the sleigh. Computing the Jacobi-Lie brackets for the fields $u_{1}, u_{2}, u_{3}$, we obtain

$$
\begin{aligned}
& {\left[u_{1}, u_{2}\right]=-\frac{k^{2}}{a} \cos k \theta u_{1}+k \sin k \theta u_{2}+u_{3},} \\
& {\left[u_{1}, u_{3}\right]=-\frac{k}{a} \cos k \theta \sin k \theta u_{1}-\cos ^{2} k \theta u_{2},} \\
& {\left[u_{2}, u_{3}\right]=\frac{k^{2}}{a^{2}} \sin ^{2} k \theta u_{1}+\frac{k}{a} \cos k \theta \sin k \theta u_{2},}
\end{aligned}
$$

which implies

$$
\begin{aligned}
& C_{12}^{1}=-\frac{k^{2}}{a} \cos k \theta, \quad C_{12}^{2}=k \sin k \theta, \quad C_{12}^{3}=1, \\
& C_{13}^{1}=-\frac{k}{a} \cos k \theta \sin k \theta, \quad C_{13}^{2}=-\cos ^{2} k \theta, \quad C_{13}^{3}=0, \\
& C_{23}^{1}=\frac{k^{2}}{a^{2}} \sin ^{2} k \theta, \quad C_{23}^{2}=\frac{k}{a} \cos k \theta \sin k \theta, \quad C_{23}^{3}=0 .
\end{aligned}
$$

Written relative to the frame $u_{1}, u_{2}, u_{3}$, the Lagrangian and the constrained Lagrangian become

$$
l(\xi)=\frac{1}{2}\left(\left(J+m a^{2}\right)\left(\xi^{1}\right)^{2}+m\left(\xi^{2}\right)^{2}+2 m a \cos k \theta \xi^{1} \xi^{3}-2 m a \sin k \theta \xi^{2} \xi^{3}+m\left(\xi^{3}\right)^{2}\right)
$$


and

$$
l_{c}(\xi)=\frac{1}{2}\left(\left(J+m a^{2}\right)\left(\xi^{1}\right)^{2}+m\left(\xi^{2}\right)^{2}\right)
$$

The constrained Hamel equations are computed to be

$$
\frac{\mathrm{d}}{\mathrm{d} t} \frac{\partial l_{c}}{\partial \xi^{1}}=0, \quad \frac{\mathrm{d}}{\mathrm{d} t} \frac{\partial l_{c}}{\partial \xi^{2}}=0 .
$$

Thus, the components of momentum relative to the frame $u_{1}, u_{2}, u_{3}$ are conserved.

\subsection{Chaplygin sleigh with an oscillator}

Here we analyse the dynamics of the Chaplygin sleigh coupled to an oscillator. We show that the phase flow is integrable, and generic invariant manifolds are two-dimensional tori.

\subsubsection{The Lagrangian, non-holonomic connection and reduced dynamics}

Consider the Chaplygin sleigh with a mass sliding along the direction of the blade. The mass is coupled to the sleigh through a spring. One end of the spring is attached to the sleigh at the contact point, the other end is attached to the mass. The spring force is zero when the mass is positioned above the contact point. See Figure 1 where the sliding mass is represented by a black dot and the blade is shown as a bold black segment.

The configuration space for this system is $\mathbb{R} \times \mathrm{SE}(2)$. This system has one shape (the distance from the mass to the contact point, $r$ ) and three group degrees of freedom.

The reduced Lagrangian $l: T \mathbb{R} \times \mathfrak{s e}(2) \rightarrow \mathbb{R}$ is given by the formula

$$
l(r, \dot{r}, \xi)=\frac{1}{2} m \dot{r}^{2}+m \dot{r} \xi^{2}+\frac{1}{2}\left(\left(J+m r^{2}\right)\left(\xi^{1}\right)^{2}+2 m r \xi^{1} \xi^{3}+(M+m)\left(\left(\xi^{2}\right)^{2}+\left(\xi^{3}\right)^{2}\right)\right)-\frac{1}{2} k r^{2},
$$

where $\xi=g^{-1} \dot{g} \in \mathfrak{s e}(2)$ and $k$ is the spring constant. The constrained reduced Lagrangian is

$$
l_{c}\left(r, \dot{r}, \xi^{1}, \xi^{2}\right)=\frac{1}{2} m \dot{r}^{2}+m \dot{r} \xi^{2}+\frac{1}{2}\left(\left(J+m r^{2}\right)\left(\xi^{1}\right)^{2}+(M+m)\left(\xi^{2}\right)^{2}\right)-\frac{1}{2} k r^{2} .
$$

The constrained reduced energy

$$
\frac{1}{2} m \dot{r}^{2}+m \dot{r} \xi^{2}+\frac{1}{2}\left(\left(J+m r^{2}\right)\left(\xi^{1}\right)^{2}+(M+m)\left(\xi^{2}\right)^{2}\right)+\frac{1}{2} k r^{2}
$$

is positive-definite, and thus the mass cannot move infinitely far from the sleigh throughout the motion.

The non-holonomic connection is

$$
\xi+\mathcal{A} \dot{r}
$$

where

$$
\mathcal{A}^{1}=0, \quad \mathcal{A}^{2}=\frac{m}{M+m}, \quad \mathcal{A}^{3}=0 .
$$

The constraint is given by the formula

$$
\Omega^{3}=0 .
$$


The reduced Lagrangian written as a function of $(r, \dot{r}, \Omega)$ becomes

$$
l(r, \dot{r}, \Omega)=\frac{1}{2} \frac{M m}{M+m} \dot{r}^{2}+\frac{1}{2}\left(\left(J+m r^{2}\right)\left(\Omega^{1}\right)^{2}+2 m r \Omega^{1} \Omega^{3}+(M+m)\left(\left(\Omega^{2}\right)^{2}+\left(\Omega^{3}\right)^{2}\right)-\frac{1}{2} k r^{2} .\right.
$$

The constrained reduced Lagrangian written as a function of $(r, \dot{r}, p)$ is

$$
l_{c}(r, \dot{r}, p)=\frac{1}{2} \frac{M m \dot{r}^{2}}{M+m}+\frac{1}{2}\left(\frac{p_{1}^{2}}{J+m r^{2}}+\frac{p_{2}^{2}}{M+m}\right)-\frac{k r^{2}}{2} .
$$

The reduced dynamics for the sleigh-mass system is computed to be

$$
\begin{aligned}
\frac{M m}{M+m} \ddot{r} & =\frac{M m r}{(M+m)\left(J+m r^{2}\right)^{2}} p_{1}^{2}-k r, \\
\dot{p}_{1} & =-\frac{m r}{(M+m)\left(J+m r^{2}\right)} p_{1} p_{2}+\frac{m^{2} r}{(M+m)\left(J+m r^{2}\right)} p_{1} \dot{r}, \\
\dot{p}_{2} & =\frac{m r}{\left(J+m r^{2}\right)^{2}} p_{1}^{2} .
\end{aligned}
$$

We now select a new frame in the Lie algebra $\mathfrak{s e}(2)$ in order to eliminate the second term in Equation (6.5). Put

$$
e_{1}=\left(\left(J+m r^{2}\right)^{-\frac{m}{2 M+m}}, 0,0\right), \quad e_{2}=(0,1,0), \quad e_{3}=(0,0,1) .
$$

The reduced Lagrangian written in this frame becomes

$$
\begin{aligned}
l(r, \dot{r}, \Omega)= & \frac{1}{2} \frac{M m}{M+m} \dot{r}^{2}+\frac{1}{2}\left(\left(J+m r^{2}\right)^{\frac{M}{M+m}}\left(\Omega^{1}\right)^{2}+(M+m)\left(\left(\Omega^{2}\right)^{2}+\left(\Omega^{3}\right)^{2}\right)\right. \\
& \left.+2 m r\left(J+m r^{2}\right)^{-\frac{M}{2 M+m}} \Omega^{1} \Omega^{3}\right)-\frac{1}{2} k r^{2} .
\end{aligned}
$$

Using Equations (4.2) and (4.3), the reduced dynamics becomes

$$
\begin{aligned}
\frac{M m}{M+m} \ddot{r} & =\frac{M m r}{(M+m)\left(J+m r^{2}\right)^{\frac{M}{M+m}+1}} p_{1}^{2}-k r, \\
\dot{p}_{1} & =-\frac{m r}{(M+m)\left(J+m r^{2}\right)} p_{1} p_{2}, \\
\dot{p}_{2} & =\frac{m r}{\left(J+m r^{2}\right)^{\frac{M}{M^{+m}+1}}} p_{1}^{2} .
\end{aligned}
$$

\subsubsection{Relative equilibria of the sleigh-mass system}

Assuming that $(r, p)=\left(r_{0}, p_{0}\right)$ is a relative equilibrium, Equation (6.8) implies $r_{0} p_{1}^{0}=0$. Thus, either $r_{0}=0$ and $p_{1}^{0}$ is an arbitrary constant, or, using (6.6), $p_{1}^{0}=0$ and $r_{0}=0$. Thus, the only relative equilibria of the sleigh-mass system are

$$
r=0, \quad p=p_{0} .
$$




\subsubsection{The discrete symmetries and integrability}

It is straightforward to see that Equations (6.6)-(6.8) are invariant with respect to the following transformations:

(i) $\left(r, p_{1}, p_{2}\right) \rightarrow\left(r,-p_{1}, p_{2}\right)$,

(ii) $\left(r, p_{1}, p_{2}\right) \rightarrow\left(-r, p_{1},-p_{2}\right)$,

(iii) $(t, r, p) \rightarrow(-t,-r, p)$,

(iv) $\left(t, r, p_{1}, p_{2}\right) \rightarrow\left(-t, r, p_{1},-p_{2}\right)$.

We now use these transformations to study some of the solutions of (6.6)-(6.8). Consider an initial condition $(r, \dot{r}, p)=\left(0, \dot{r}_{0}, p_{0}\right)$. Then the $r$-component of the solution subject to this initial condition is odd, and the $p$-component is even. Indeed, let

$$
(r(t), p(t)), \quad t>0
$$

be the part of this solution for $t>0$. Then

$$
(-r(-t), p(-t)), \quad t<0
$$

is also a solution. This follows from the invariance of Equations (6.6)-(6.8) with respect to transformation (iii). Using the formula

$$
\left.\frac{\mathrm{d} r(t)}{\mathrm{d} t}\right|_{t=0}=\left.\frac{\mathrm{d}(-r(-t))}{\mathrm{d} t}\right|_{t=0}
$$

we conclude that (6.9) and (6.10) satisfy the same initial condition and thus represent the forward in time and the backward in time branches of a the same solution. Thus, $r(-t)=-r(t)$ and $p(-t)=p(t)$.

Next, $p_{1}(t)=0$ implies that $p_{2}(t)=$ const and $r(t)$ satisfies the equation

$$
\frac{M m}{M+m} \ddot{r}=-k r
$$

and thus Equations (6.6)-(6.8) have periodic solutions

$$
r(t)=A \cos \omega t+B \sin \omega t, \quad p_{1}=0, p_{2}=C,
$$

where $A, B$, and $C$ are arbitrary constants and $\omega=\sqrt{k(M+m) / M m}$.

Without loss of generality, we set $A=0$ and consider periodic solutions

$$
r(t)=\dot{r}_{0} / \omega \sin \omega t, \quad p_{1}=0, p_{2}=p_{2}^{0},
$$

which correspond to the initial conditions

$$
r(0)=0, \quad \dot{r}(0)=\dot{r}_{0}, \quad p_{1}(0)=0, \quad p_{2}(0)=p_{2}^{0} .
$$

We now perturb solutions (6.11) by setting

$$
r(0)=0, \quad \dot{r}(0)=\dot{r}_{0}, \quad p_{1}(0)=p_{1}^{0}, \quad p_{2}(0)=p_{2}^{0} .
$$


Assuming that $p_{1}^{0}$ is small and using a continuity argument, there exists $\tau=\tau_{p, r_{0}}>0$ such that

$$
r\left(\tau_{p, \dot{r}_{0}}\right)=0
$$

for solutions subject to initial conditions (6.12). That is, the $r$-component is $2 \tau$-periodic if $p_{1}^{0}$ is sufficiently small.

Using Equation (6.6) and periodicity of $r(t)$, we conclude that $p_{1}$ is $2 \tau$-periodic as well. Equation (6.7) then implies that $p_{2}(t)$ is also $2 \tau$-periodic. Thus, the reduced dynamics is integrable in an open subset of the reduced phase space. The invariant tori are one-dimensional, and the reduced flow is periodic. A generic periodic trajectory in the direct product of the shape and momentum spaces is shown in Figure 3.

Using the quasi-periodic reconstruction theorem $[23,24]$, we obtain the following theorem.

Theorem 6.1: Generic trajectories of the coupled sleigh-oscillator system in the full phase space are quasi-periodic motions on two-dimensional invariant tori.

Typical trajectories of the contact point of the sleigh with the plane are shown in Figure 4. The symmetry observed in these trajectories follows from the existence, for each group trajectory $g(t)$, of a group element $h$ such that $g(t+2 \tau) \equiv h g(t)$, where $2 \tau$ is the

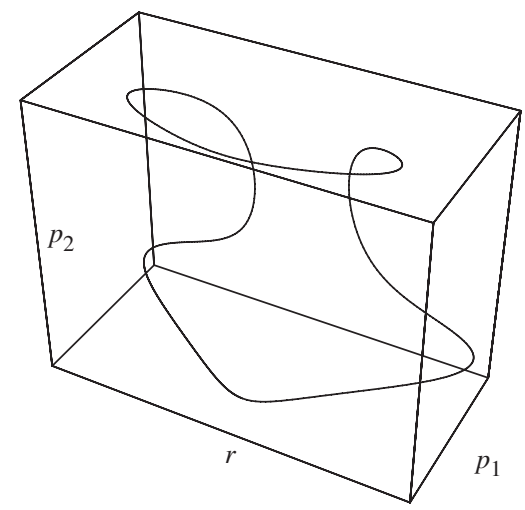

Figure 3. A reduced trajectory of the sleigh-mass system.
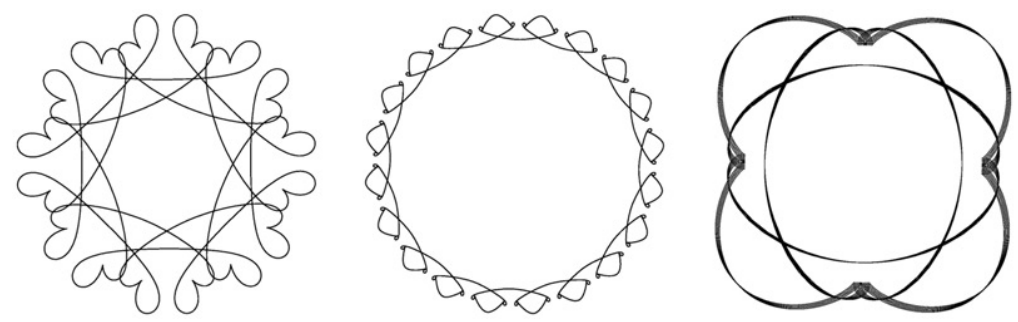

Figure 4. Trajectories of the contact point of the blade for various initial states. 
period of the corresponding reduced dynamics. It would be interesting to see if the technique of pattern evocation $[25,26]$ extends to the current circumstances.

\subsection{Asymptotic behaviour of momentum integrals}

Assuming that the only non-zero term in the right-hand side of the momentum equation (4.7) is $\left\langle p, \mathbf{i}_{i} \tau\right\rangle$, Theorem 4.4 and Corollary 4.5 give sufficient conditions for existence of $m$ independent momentum integrals $\left\langle p, \eta_{a}(r)\right\rangle, a=1, \ldots, m$. The fields $\eta_{a}(r) \in \mathfrak{b}^{\mathcal{S}}$ satisfy Equation (4.12), their existence follows from the integrability of the distribution associated with the form $\tau$ (see Theorem 4.4 for details). In the coordinate form, Equation (4.12) reads

$$
\mathrm{d} \eta^{b}=-\mathcal{D}_{a \alpha}^{b}(r) \eta^{a} \mathrm{~d} r^{\alpha},
$$

where the quantities $\mathcal{D}_{a \alpha}^{b}(r)$ are defined by formula (4.11).

The properties of distribution (6.13) resemble the properties of linear homogeneous systems of differential equations as the coefficients of the differential forms in (6.13) are linear functions in the components of $\eta$. It is straightforward to show that the space of integral manifolds of distribution (6.13) has the structure of an $m$-dimensional vector space.

Definition 6.2: The Wronskian of the $m$ integral manifolds $\eta=\eta_{a}(r), a=1, \ldots, m$, is the determinant

$$
W(r)=\operatorname{det}\left[\begin{array}{ccc}
\eta_{1}^{1}(r) & \ldots & \eta_{m}^{1}(r) \\
\vdots & & \vdots \\
\eta_{1}^{m}(r) & \ldots & \eta_{m}^{m}(r)
\end{array}\right] .
$$

The properties of the Wronskian of the system of invariant manifolds of (6.13) are similar to those of the Wronskian of the solutions of a system of linear ordinary differential equations.

Theorem 6.3: The Wronskian satisfies the equation

$$
\mathrm{d} \ln W=-\operatorname{Tr} \tau:=-\mathcal{D}_{a \alpha}^{a}(r) \mathrm{d} r^{\alpha} .
$$

Proof: Taking into account (6.13) and (6.14), we obtain

$$
\frac{\partial W}{\partial r^{\alpha}}=-W \mathcal{D}_{a \alpha}^{a} .
$$

Therefore,

$$
\mathrm{d} W=\frac{\partial W}{\partial r^{\alpha}} \mathrm{d} r^{\alpha}=-W \mathcal{D}_{a \alpha}^{a} \mathrm{~d} r^{\alpha}=-W \operatorname{Tr} \tau,
$$

which is equivalent to formula (6.15).

Corollary 6.4: The Wronskian can be evaluated by the formula

$$
W(r)=W\left(r_{0}\right) \exp \int_{r_{0}}^{r} \operatorname{Tr} \tau .
$$

The integral in (6.16) is independent of path from $r_{0}$ to $r$. 
Formula (6.16) allows one to obtain the Wronskian explicitly in the multi-dimentional shape space setting. This result can be used to study the reduced energy levels even if the momentum integrals cannot be effectively computed as in [40]. We demonstrate this in the following section for the rolling disk.

\subsection{The rolling falling disk}

To illustrate the results of Section 4.3 and Section 6.4, consider a disk rolling without sliding on a horizontal plane (Figure 5). The configuration space for this system is $(-\pi / 2, \pi / 2) \times \mathrm{SO}(2) \times \mathrm{SE}(2)$. The coordinates on the configuration space are denoted $(\theta, \psi, \phi, x, y)$. As the figure indicates, we denote the coordinates of contact of the disk in the $x y$-plane by $(x, y)$, and let $\theta, \phi$, and $\psi$ represent the angle between the plane of the disk and the vertical axis, the 'heading angle' of the disk, and 'self-rotation' angle of the disk, respectively. Denote the mass, the radius and the moments of inertia of the disk by $m$, $R, A$ and $B$, respectively. The Lagrangian is given by the kinetic minus potential energies:

$$
\begin{aligned}
L= & \frac{m}{2}\left[\left(\zeta^{1}-R(\dot{\phi} \sin \theta+\dot{\psi})\right)^{2}+\left(\zeta^{2}\right)^{2} \sin ^{2} \theta+\left(\zeta^{2} \cos \theta+R \dot{\theta}\right)^{2}\right] \\
& +\frac{1}{2}\left[A\left(\dot{\theta}^{2}+\dot{\phi}^{2} \cos ^{2} \theta\right)+B(\dot{\phi} \sin \theta+\dot{\psi})^{2}\right]-m g R \cos \theta,
\end{aligned}
$$

where $\zeta^{1}=\dot{x} \cos \phi+\dot{y} \sin \phi+R \dot{\psi}$ and $\zeta^{2}=-\dot{x} \sin \phi+\dot{y} \cos \phi$, while the constraints are given by

$$
\dot{x}=-\dot{\psi} R \cos \phi, \quad \dot{y}=-\dot{\psi} R \sin \phi .
$$

Note that the constraints may also be written as $\zeta^{1}=0, \zeta^{2}=0$.

This system is invariant under the action of the group $G=\mathrm{SO}(2) \times \mathrm{SE}(2)$; the action by the group element $(\alpha, \beta, a, b) \in \mathrm{SO}(2) \times \mathrm{SE}(2)$ is given by

$$
(\theta, \psi, \phi, x, y) \mapsto(\theta, \psi+\alpha, \phi+\beta, x \cos \beta-y \sin \beta+a, x \sin \beta+y \cos \beta+b) .
$$

The fibres of the constraint distribution $\mathcal{D}_{q}$ are spanned by the vector fields $\partial_{\theta}, \partial_{\phi}$, and $-R \cos \phi \partial_{x}-R \sin \phi \partial_{y}+\partial_{\psi}$. Therefore,

$$
\mathcal{S}_{q}=\mathcal{D}_{q} \cap T_{q} \operatorname{Orb}(q)=\operatorname{span}\left(\partial_{\phi},-R \cos \phi \partial_{x}-R \sin \phi \partial_{y}+\partial_{\psi}\right) .
$$

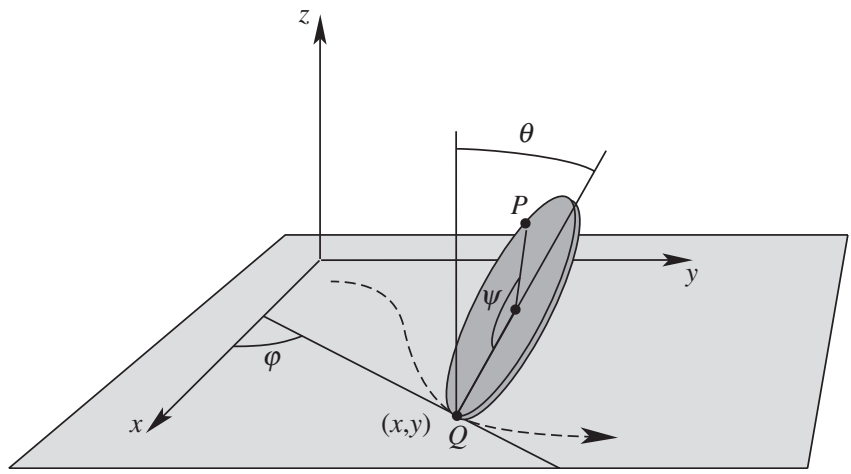

Figure 5. The geometry for the rolling disk. 
We select the basis of the subspace $\mathfrak{b}^{\mathcal{S}} \subset \mathfrak{s p}(2) \times \mathfrak{s e}(2)$ to be

$$
e_{1}=(1,0, R, 0) \text { and } e_{2}=\left(-\tan \theta, \cos ^{-1} \theta,-R \tan \theta, 0\right) .
$$

The two components of the non-holonomic momentum relative to $e_{1}$ and $e_{2}$ are

$$
\begin{aligned}
& p_{1}=A \dot{\phi} \cos ^{2} \theta+\left(m R^{2}+B\right)(\dot{\phi} \sin \theta+\dot{\psi}) \sin \theta, \\
& p_{2}=-A \dot{\phi} \cos ^{2} \theta \sin \theta+\left(m R^{2}+B\right)(\dot{\phi} \sin \theta+\dot{\psi}) \cos ^{2} \theta .
\end{aligned}
$$

The momentum equations for the falling disk are computed to be

$$
\dot{p}_{1}=\left(\tan \theta p_{1}-\frac{B}{m R^{2}+B} p_{2}\right) \dot{\theta}, \quad \dot{p}_{2}=-\frac{m R^{2}}{A} p_{1} \dot{\theta} .
$$

According to Corollary 4.5 , there exists a basis $\eta_{1}(\theta), \eta_{2}(\theta)$ of the subspace $\mathfrak{b}^{\mathcal{S}}$ such that the components $\left\langle p, \eta_{1}(\theta)\right\rangle$ and $\left\langle p, \eta_{2}(\theta)\right\rangle$ of non-holonomic momentum are constants of motion.

We write the momentum levels as $c_{1}$ and $c_{2}$, i.e.

$$
\left\langle p, \eta_{1}(\theta)\right\rangle=c_{1}, \quad\left\langle p, \eta_{2}(\theta)\right\rangle=c_{2} .
$$

Given the momentum levels $c_{1}$ and $c_{2}$, Equations in (6.17) define $p_{1}$ and $p_{2}$ as functions of $\theta$. The vector fields $\eta_{1}(\theta)$ and $\eta_{2}(\theta)$ form the fundamental solution of the linear system

$$
\frac{\mathrm{d} \eta^{1}}{\mathrm{~d} \theta}=-\tan \theta \eta^{1}+\frac{m R^{2}}{A} \eta^{2}, \quad \frac{\mathrm{d} \eta^{2}}{\mathrm{~d} \theta}=\frac{B}{m R^{2}+B} \eta^{2} .
$$

It is possible to write the solutions of system (6.18) in terms of the hypergeometric function. See [27-29] for details. For additional work and history of the falling disk see $[16,30,31]$.

Kolesnikov [32] proves that the disk does not fall, i.e. the absolute value of the tilt does not reach the value of $\pi / 2$ in finite time, for all but a codimension one set of initial conditions. It is interesting that the proof does not use the explicit formulae for the fields $\eta_{1}(\theta)$ and $\eta_{2}(\theta)$.

Indeed, the boundaries for the tilt of the disk $\theta$ are determined by the inequality $U_{c}(\theta) \leq h$, where $h$ is the energy level and

$$
U_{c}(\theta)=\frac{1}{2}\left(p_{1}^{2}(\theta) / A+p_{2}^{2}(\theta) /\left(m R^{2}+B\right)\right)+m g R \cos \theta
$$

is the amended potential restricted to the momentum level (6.17). The crucial step in the proof is to show that $\lim _{\theta \rightarrow \pm \pi / 2} U_{c}(\theta)=\infty$ for most initial conditions. As in Section 6.4, consider the Wronskian

$$
W(\theta)=\operatorname{det}\left[\begin{array}{ll}
\eta_{1}^{1}(\theta) & \eta_{2}^{1}(\theta) \\
\eta_{1}^{2}(\theta) & \eta_{2}^{2}(\theta)
\end{array}\right] .
$$

By Liouville's formula (which is a special case of (6.16))

$$
W(\theta)=W(0) \exp \int_{0}^{\theta}-\tan \theta \mathrm{d} \theta=W(0) \cos \theta .
$$


Therefore, $W(0) \rightarrow 0$ as $\theta \rightarrow \pm \pi / 2$. Formulae (6.17) thus imply that the absolute value of at least one of the momentum components $p_{1}(\theta), p_{2}(\theta)$ approaches infinity as $\theta \rightarrow \pm \pi / 2$, and so does $U_{c}(\theta)$ for all but a codimension one subset of $c=\left(c_{1}, c_{2}\right)$.

\section{Conclusions}

In this article, we studied the use of quasivelocities and Hamel equations in the dynamics of non-holonomic systems with symmetry. In particular, we discussed the importance of the choice of a basis of the tangent bundle of the configuration space in studying momentum conservation and integrability of non-holonomic systems. We showed that these ideas could be very helpful in analysing specific examples such as the sleigh-mass system and the falling disk.

In future work, we intend to extend some of these ideas to infinite-dimensional systems, to the analysis of control of non-holonomic systems, and to the dynamics of discrete non-holonomic systems, both free and controlled.

\section{Acknowledgements}

We thank Yakov Berchenko-Kogan, Hernán Cendra, Michael Coleman, Brent Gillespie, P.S. Krishnaprasad, Jared Maruskin, Richard Murray, Tudor Ratiu, and Andy Ruina for numerous discussions, and the referees for helpful suggestions. The research of AMB was supported by NSF grants DMS-0604307 and CMS-0408542. The research of JEM was partially supported by AFOSR Contract FA9550-08-1-0173. The research of DVZ was partially supported by NSF grants DMS0306017 and DMS-0604108.

\section{Notes}

1. In certain cases, some or all of $u_{i}$ can be chosen to be global vector fields on $Q$.

2. If $Q$ is a Lie group, this formula is derived in [18].

3. Euler wrote the three components of the equations $\frac{\mathrm{d}}{\mathrm{d} t} \frac{\partial l}{\partial \xi}=\frac{\partial l}{\partial \xi} \times \xi$, with explicit formulae substituted for $\frac{\partial l}{\partial \xi}$, where $l$ is the reduced rigid body Lagrangian.

4. Constraints are non-holonomic if and only if they cannot be rewritten as position constraints.

5. If $\mathcal{S}_{q}=\{0\}$, a set of non-holonomic constraints is said to be purely kinematic.

6. Recall that the components $\mathcal{A}_{\alpha}^{a^{\prime}}$ are defined by the constraints.

7. A Lie group is said to be unimodular if it has a bilaterally invariant measure.

\section{References}

[1] J.I. Neimark and N.A. Fufaev, Dynamics of Nonholonomic Systems, Vol. 33, Translations of Mathematical Monographs, AMS, Providence, Rhode Island, 1972.

[2] D.T. Greenwood, Advanced Dynamics, Cambridge University Press, Cambridge, 2003.

[3] L. Euler, Decouverte d'un nouveau principe de Mecanique, Mém. Acad. Sci. Berlin 6 (1752), pp. 185-217.

[4] J.L. Lagrange, Mécanique Analytique, Chez la Veuve Desaint, Paris, 1788.

[5] H. Poincaré, Sur une forme nouvelle des équations de la mécanique, CR Acad. Sci. 132 (1901), pp. $369-371$.

[6] J.E. Marsden and T.S. Ratiu, Introduction to Mechanics and Symmetry 1st ed. 1994, 2nd ed., 1999, Vol. 17, Springer-Verlag, Texts in Applied Mathematics, New York, 1999. 
[7] E.J. Routh, Treatise on the Dynamics of a System of Rigid Bodies, MacMillan, London, 1860.

[8] A.P. Markeev, Dynamics of a Body Being Contiguous to a Rigid Surface, Nauka, Moscow, 1992 (In Russian).

[9] G. Hamel, Die Lagrange-Eulersche Gleichungen der Mechanik, Z. Math. Phys. 50 (1904), pp. 1-57.

[10] A.M. Bloch, P.S. Krishnaprasad, J.E. Marsden, and R. Murray, Nonholonomic mechanical systems with symmetry, Arch. Rat. Mech. An. 136 (1996), pp. 21-99.

[11] H. Cendra, J.E. Marsden, and T.S. Ratiu, Lagrangian reduction by stages, Vol. 152, Memoirs of the American Mathematical Society, Providence, R.I., 2001.

[12] D.V. Zenkov, A.M. Bloch, and J.E. Marsden, The energy momentum method for the stability of nonholonomic systems, Dyn. Stab. Syst. 13 (1998), pp. 123-166.

[13] A. Ruina, Non-holonomic stability aspects of piecewise nonholonomic systems, Rep. Math. Phy. 42 (1998), pp. 91-100.

[14] A.M. Bloch, Asymptotic Hamiltonian dynamics: The Toda lattice, the three wave interaction and the non-holonomic Chaplygin sleigh, Physica D 141 (2000), pp. 297-315.

[15] D.V. Zenkov and A.M. Bloch, Invariant measures of nonholonomic flows with internal degrees of freedom, Nonlinearity 16 (2003), pp. 1793-1807.

[16] A.M. Bloch, Nonholonomic Mechanics and Control, Interdisciplinary Applied Mathematics, Springer-Verlag, New York, 2003.

[17] J.F. Cariñena, J.M. Nunes da Costa, and P.S. Santos, Quasicoordinates from the point of view of Lie algebroid structures, J. Phys. A: Math. Theor. 40 (2007), pp. 10031-10048.

[18] A.M. Bloch, P.S. Krishnaprasad, J.E. Marsden, and T.S. Ratiu, The Euler-Poincaré equations and double bracket dissipation, Comm. Math. Phys. 175 (1996), pp. 1-42.

[19] H. Cendra, J.E. Marsden, and T.S. Ratiu, Geometric mechanics, Lagrangian reduction and nonholonomic systems, In Mathematics Unlimited-2001 and Beyond, B. Enquist and W. Schmid, eds., Springer-Verlag, New York, 2001, pp. 221-273.

[20] D.V. Zenkov, Linear conservation laws of nonholonomic systems with symmetry, Disc. Cont. Dyn. Syst. (extended volume) (2003), pp. 963-972.

[21] V.V. Kozlov, Invariant measures of the Euler-Poincaré equations on Lie algebras, Functional Anal. Appl. 22 (1988), pp. 69-70.

[22] B. Jovanović, Nonholonomic geodesic flows on lie groups and the integrable Suslov problem on SO(4), J. Phys. A: Math. Gen. 31 (1998), pp. 1415-1422.

[23] P. Ashwin and I. Melbourne, Noncompact drift for relative equilibria and relative periodic orbits, Nonlinearity 10 (1997), pp. 595-616.

[24] M.J. Field, Equivariant dynamical systems, Trans. Am. Math. Soc. 259 (1980), pp. 185-205.

[25] J.E. Marsden and J. Scheurle, Pattern Evocation and Geometric Phases in Mechanical Systems with Symmetry, Dynam. Stabil. Syst. 10 (1995), pp. 315-338.

[26] J.E. Marsden, J. Scheurle, and J. Wendlandt, Visualization of orbits and pattern evocation for the double spherical pendulum, In ICIAM 95: Mathematical Research, Vol. 87, K. Kirchgssner, O. Mahrenholtz, and R. Mennicken, eds., Academie Verlag, Berlin, 1996, pp. 213-232.

[27] P. Appel, Sur l'intégration des équations du mouvement d'un corps pesant de révolution roulant par une arête circulaire sur un plan horizontal; cas particulier du cerceau, Rendiconti del circolo matematico di Palermo 14 (1900), pp. 1-6.

[28] S.A. Chaplygin, On the Motion of a Heavy Body of Revolution on a Horizontal Plane (in Russian), Phys. Sec. Imperial Soc. Friends Phys., Anthropol. Ethnograph., Moscow 9 (1897), pp. $10-16$.

[29] D. Korteweg, Über eine ziemlich verbreitete unrichtige Behandlungsweise eines Problemes der rollenden Bewegung und insbesondere über kleine rollende Schwingungen um eine Gleichgewichtslage, Nieuw Archiefvoor Wiskunde 4 (1899), pp. 130-155.

[30] A. Vierkandt, Über gleitende und rollende Bewegung, Monatshefte der Math. und Phys. III (1892), pp. 31-54. 
[31] R. Cushman, J. Hermans, and D. Kemppainen, The rolling disc, In Nonlinear Dynamical Systems and Chaos (Groningen, 1995), Vol. 19, H.W. Broer, S.A. van Gils, I. Hoveijn, and F. Takens, eds., Birkhauser, Basel, Boston, MA, 1996, pp. 21-60, Progress in Nonlinear Differential Equations and their Applications.

[32] S.N. Kolesnikov, On a disk rolling on a horizontal plane, Vestn. Mosk. Univ. 2 (1985), pp. 55-60.

[33] L. Bates and J. Sniatycki, Nonholonomic reduction, Rep. Math. Phys. 32 (1993), pp. 99-115.

[34] S.A. Chaplygin, On the rolling of a sphere on a horizontal plane, Mat. Sbornik XXIV (1903), pp. 139-168 (in Russian).

[35] S.A. Chaplygin, Analysis of the Dynamics of Nonholonomic Systems, Classical Natural Sciences, GITTL, Moscow, 1949.

[36] A.N. Kolmogorov, On dynamical systems with an integral invariant on the torus (in Russian), Doklady Akad. Nauk SSSR 93 (1953), pp. 763-766.

[37] V.V. Kozlov, On the integration theory of the equations in nonholonomic mechanics, Adv. Mech. 8(3) (1985), pp. 86-107.

[38] V.V. Kozlov, Symmetries, Topology, and Resonanses in Hamiltonian Mechanics, SpringerVerlag, Berlin, 1995.

[39] A.P. Veselov and L.E. Veselova, Integrable nonholonomic systems on Lie groups, Math. Notes 44 (1988), pp. 810-819.

[40] D.V. Zenkov, The geometry of the Routh problem, J. Nonlinear Sci. 5 (1995), pp. 503-519. 